\title{
Transformations of Visuospatial Images
}

\author{
Jeffrey M. Zacks \\ Pascale Michelon \\ Washington University in Saint Louis
}

Transformations of visuospatial mental images are important for action, navigation, and reasoning. They depend on representations in multiple spatial reference frames, implemented in the posterior parietal cortex and other brain regions. The multiple systems framework proposes that different transformations can be distinguished in terms of which spatial reference frame is updated. In an object-based transformation, the reference frame of an object moves relative to those of the observer and the environment. In a perspective transformation, the observer's egocentric reference frame moves relative to those of the environment and of salient objects. These two types of spatial reference frame updating rely on distinct neural processing resources in the parietal, occipital, and temporal cortex. They are characterized by different behavioral patterns and unique individual differences. Both object-based transformations and perspective transformations interact with posterior frontal cortical regions subserving the simulation of body movements. These interactions indicate that multiple systems coordinate to support everyday spatial problem solving.

Key Words: imagery, visuospatial, mental rotation

$\mathbf{H}_{\mathrm{e}}$ Imagine you are standing in front of a pedestal facing a bust of William James. Imagine now that you have walked around the pedestal and are now viewing the bust from the side. Now, imagine that a motor is turning the bust, allowing you to view it from a range of angles. Finally, imagine that you reach out, pick up the bust, and turn it upside-down to look at the bottom."

Most people report that they can follow such instructions, and doing so often results in phenomenal experiences that are vivid and faithful to reality-though the degree that this is the case varies among individuals (Isaac and Marks, 1994). The experiences evoked by instructions such as these are transformations of visuospatial images. They may not require explicit intentions to produce: Humans and other animals appear to construct and transform visuospatial representations to solve a range of everyday reasoning problems, including navigation, the making and using of tools, and construction. But what is the computational and neurophysiological nature of the representations involved? How are those representations transformed during explicit and implicit visuospatial imagery?

There is now a body of theory and evidence speaking to each of these questions. The goals of this article are, first, to introduce a framework for thinking about visuospatial image transformations, and, second, to interpret the available data within that framework to provide current best answers to both questions. To do so, we first provide an overview of the phenomena constituting visuospatial imagery and then describe a framework for relating image transformations to the representations on which they operate. The rest of the article characterizes different types of image transformations and the relations among them. The article's major empirical claim is that two sorts of visuospatial transformations can be dissociated: transformations in which the representations of individual objects are updated relative to other spatial representations and transformations in which one's personal perspective is updated. These visuospatial transformations are characterized by different patterns of behavioral performance, different neural correlates, and different psychometric properties.

\footnotetext{
Authors' Note: We would like to thank Ty Fagan for his assistance with the meta-analysis. We also thank Sarah Creem-Regehr, Nora Newcombe, Amy Shelton, Barbara Tversky, and Maryjane Wraga, whose thoughtful comments were extremely helpful in revising the article. The research of our laboratory described here was supported in part by the McDonnell Center for Higher Brain Function.

Behavioral and Cognitive Neuroscience Reviews

Volume 4 Number 2, June 2005 96-118

DOI: $10.1177 / 1534582305281085$

(C) 2005 Sage Publications
} 


\section{VISUOSPATIAL MENTAL IMAGERY}

Visuospatial imagery received steady empirical attention at least since the studies of Francis Galton in the 1800s (Miller, 1962, pp. 138-139). In those initial studies, it was widely assumed that visuospatial images were represented as something like "pictures in the head." Then, the rise of behaviorism made the study of mentalistic phenomena such as imagery intensely unpopular in mainstream experimental psychology (Schultz, 1975), though students of the Gestalt school of psychology continued some studies of visuospatial imagery (Köhler, 1929). Visuospatial mental imagery reentered the psychological mainstream with the cognitive revolution in the 1960s and 70s-and along with it came the computer metaphor for the mind (Gardner, 1985, chap. 3). On this view, the brain and the mind function as a generalpurpose computer, and the representations that underlie cognition are domain-general symbols that are akin to the symbols in a natural language or computer programming language. This view led to a question regarding visuospatial imagery, which had not arisen during the early history of psychology: Are the representations underlying imagery really like pictures in the head, or are they really like expressions in a language?

This question has been debated for more than three decades (Pylyshyn, 1973; Kosslyn and Pomerantz, 1977; Kosslyn, 1980, 1981; Pylyshyn, 1981; Finke, 1985; Finke and Shepard, 1986; Kosslyn, 1994; Pylyshyn, 2001, 2002, 2003). The central question is whether the representations that underlie mental imagery are analog and isomorphic to the situations they represent (the analog view) or discrete and language-like, with no more isomorphism to their referents than the word $\operatorname{dog}$ has to a real dog (the propositional view). This extended debate has had the salutary effect of forcing mental imagery researchers to provide explicit mechanistic accounts of visuospatial image representations and the transformations that operate on them. In our view, the preponderance of the evidence weighs with the analog view. However, it is the details of the mechanistic accounts that matter for present purposes rather than the answer to the question of whether visuospatial mental images are better thought of as analog or propositional, and there is broad consensus about these accounts. The current consensus view among mental imagery theorists subscribing to the analog view can be summarized in the following two claims (Kosslyn, 1994):

1. Visuospatial images are represented by neural-processing systems in the cerebral cortex that also subserve perception. As we will lay out in following sections, the extent of overlap between perception and imagery is a matter of ongoing research and debate.
2. Whereas visuospatial perception is driven largely by bottom-up sensory input, visuospatial imagery is driven largely by top-down knowledge-based activation. Visuospatial images may be created and updated based on representations from memory, from linguistic input, or from inferences.

This broad characterization leaves some important questions about visuospatial reasoning unanswered. What is the nature of the spatial representations that are formed in perception and imagery? How are these representations transformed when people imagine changes to the spatial configuration of themselves and their environment? The following section develops a theoretical framework for answering these two questions, and the succeeding sections review data that bear on this framework.

\section{SPATIAL REFERENCE FRAMES AND MENTAL TRANSFORMATIONS}

\section{Three Types of Spatial Reference Frames}

Spatial representations locate things. To locate something, it is necessary to specify an origin and one or more axes. These factors constitute a reference frame. (It is not, however, necessary that the reference frame satisfy the requirements of Euclidean geometry. The axes need not be orthogonal and need not represent ratio-level numerical information. For example, when locating buildings on an imperfect street grid, such as "Five blocks west of City Hall on Third Street," the axes provided by the street grid may be somewhat skewed, and the block-to-block distances may not be equal.) Three major classes of reference frames that are relevant for human cognition can be distinguished: object-based reference frames, which are defined relative to external objects; egocentric reference frames, which are defined relative to the self; and environmental reference frames, which are defined relative to some fixed feature or features of the environment. (Our characterization of spatial reference frames is heavily indebted to a thoughtful chapter on this subject by McCloskey, 2001. Levinson, 1996, also has written thoughtfully about these issues from a psycholinguistic perspective.) There is ample evidence that the human nervous system makes use of all three of these sorts of reference frames.

Object-based reference frames locate things relative to one or more axes defined with respect to a particular object. Object-based reference frames tend to have one or three dimensions. For example, a pencil may be represented as having a major (up-down) axis running from the eraser to the tip, but having no front-back or leftright axis. A teapot may be represented as having an updown axis, a front-back axis, and a left-right axis. In per- 
ception, people appear to rapidly and automatically assign a major axis and hence a top to objects, which plays a pervasive role in their spatial reasoning (Rock, 1974). An object-based reference frame provides a means to characterize the relationship between the parts of an object independent of the object's location in the world. ${ }^{1}$ An object-based reference frame also can be used to locate an object relative to another other object (e.g., "behind the piano").

Egocentric reference frames locate things relative to axes defined with respect to the self. For coarse-grained spatial relations, humans appear to pervasively use an egocentric reference frame with three axes: up-down, front-back, and left-right. Under most circumstances, people can quickly and accurately locate nearby objects relative to this reference frame, with up-down judgments being fastest and left-right judgments being slowest (Franklin and Tversky, 1990). For such judgments, it is not necessary to specify exactly where on the body the origin of the reference frame is placed. However, there is extensive evidence that the nervous system implements a number of finer-grained egocentric reference frames that code the location of things relative to different body parts. (For a review, see Colby, 1998.) In the monkey, the lateral intraparietal area contains representations in eyecentered reference frames, and the adjacent ventral intraparietal area contains representations in headcentered reference frames. In eye-centered reference frames, the location of objects changes every time the eyes move. In head-centered frames, the location of objects is invariant over eye movements but changes when the head moves. Egocentric reference frames are critical for the control of action; to reach out and grab something, it is necessary to locate it in multiple egocentric reference frames. For controlling reaching movements, a series of transformations, likely implemented in the superior parietal lobule and posterior frontal cortex, map from eye-centered coordinates to hand-centered coordinates, which code the location of objects relative to the hand (Graziano and Gross, 1994; Snyder, Batista, and Andersen, 1997). For navigation as well, it is necessary to locate targets in egocentric reference frames. To walk to a drinking fountain, one must represent its distance and direction relative to the self.

Environmental reference frames locate things relative to axes defined with respect to a fixed space, such as the principal axes of a rectangular room or the cardinal directions in geography (north, south, east, west). In the neuropsychological literature, these reference frames are usually called allocentric reference frames. When people enter a rectangular room, they appear to assign it a stable set of axes (Shelton and McNamara, 2001). If the viewer is initially aligned with the walls, the walls are usually taken as the axes. The configuration of objects in an environment also can establish an environmental reference frame (Mou and McNamara, 2002; Mou, McNamara, Valiquette, and Rump, 2004). The layout of large spaces and the locations of buildings and objects may be coded in environmental reference frames by cells in the hippocampus. The properties of such cells have been studied extensively in rodents (O'Keefe and Nadel, 1978) and more recently in humans (Ekstrom et al., 2003). The role of the hippocampus in human long-term memory for locations remains controversial (Teng and Squire, 1999; Rosenbaum et al., 2000). One recent proposal is that environmental location is coded by the spatial distribution of hippocampal activity, but other features of episodic memory are coded by other neural properties including the rate of firing (Leutgeb et al., 2005).

\section{Transformations of Spatial Reference Frames}

With this classification of spatial reference frames in mind, we are in a position to consider the different types of spatial transformations that are possible. At any moment, the origins of each reference frame maintained by an organism are located relative to the other reference frames. (The relationships may not be explicit, and need not be accurate, but they are a necessary consequence of the fact that the origin of a reference frame is itself a spatial location that can be specified relative to each reference frame.) The simplest classes of spatial transformations are those in which the location or orientation of one reference frame is updated relative to the other reference frames, but those other reference frames maintain their relationship to each other. More complex transformations can be constructed by combining these simple transformations. Thus, if a toy car rolls past an observer across a room, the object-centered reference frame of the car moves relative to the egocentric reference frames of the observer and the environmental reference frame. We will refer to such transformations as object-based transformations. If the observer moves toward the toy car while maintaining a steady posture and eye position, the observer's egocentric reference frames move relative to the reference frames of the car and the room. This reference frame could be called an egocentric-based spatial transformation. However, we have already seen that there are a number of egocentric reference frames maintained by the nervous system at any one time, so this characterization is too coarse. When considering visuospatial imagery, it is useful to focus on two classes of egocentric reference frame: those that represent things from the observer's visual perspective (eye-centered or head-centered reference frames) and those that represent things relative to the observer's effectors. Thus, if the observer turns to face the new location of the toy car, the observer's eye-centered reference 
TABLE 1: Reference Frames and Their Relationship to Spatial Transformations

\begin{tabular}{llll}
\hline & & \multicolumn{2}{c}{ Reference Frame That Moves } \\
\cline { 3 - 4 } & & Ebject-Centered & Egocentric \\
\cline { 3 - 4 } Spatial Transformation & Object-based & $\checkmark$ & \\
& Perspective & & $\checkmark$ \\
& Effector-based & $\checkmark$ & $\checkmark$ \\
& Not observed & & $\checkmark$ \\
\hline
\end{tabular}

frame is updated relative to the object-centered and environmental reference frames. We will refer to this reference frame as a perspective transformation ${ }^{2}$. If the observer reaches out toward the toy car, this movement updates the observer's effector-centered (hand-centered) reference frame relative to the object-centered and environmental reference frames, which updates the relationships between an effector-centered reference frame and the other reference frames-an effector-based transformation. Finally, if the floor, walls, and ceiling of the room were to drop $3 \mathrm{ft}$, leaving the toy car and the observer hanging in space, this event would update the environmental reference frame relative to the object-centered and egocentric reference frames. However, as is suggested by the example, these sorts of transformations rarely, if ever, occur in normal terrestrial experience. They do not appear to play a substantive role in visuospatial imagery. Therefore, they will not be discussed further.

In short, three types of spatial reference frames are critical for representing humans' interactions with their spatial environment: object-centered, egocentric, and environmental. Egocentric reference frames can be broken down into those centered on the head, on an effector, or others. Updating an object-based, headcentered, or effector-centered representation gives rise to one of three basic spatial transformations; more complex transformations can be derived by combining the basic ones. The relationship between these reference frames and transformations is summarized in Table 1. (See Wraga, Creem, and Proffitt, 1999 for a similar analysis of a subset of these relations.)

Object-based transformations, perspective transformations, and effector-based transformations can be imagined as well as performed. As will be seen in the following sections, imagined object-based transformations encompass processes that have been studied under the rubrics of mental rotation and mental translation. Imagined perspective transformations encompass transformations evoked in viewer rotation or translation tasks and some kinds of perspective-taking tasks. Imagined effector-based transformations occur during action planning and rehearsal. They are at the core of the simulation of body movements during motor imagery (Michelon, Vettel, and Zacks, manuscript submitted for publication).

\section{The Multiple Systems Framework}

We hypothesize that normal spatial reasoning ability requires the integrated functioning of several distinct neural resources. First, it requires intact representations of location in object-centered, egocentric, and effectorcentered reference frames. These reference frames may be implemented by anatomically distinct neural substrates. Second, it requires input resources that recode location information from low-level sensory representations into these reference frames. Third, it requires output resources that feed location information in each reference frame to systems that subserve activities such as language production, movement production, and longterm memory. Finally, we hypothesize that each type of imagined reference frame transformation requires unique processing resources. In short, we hypothesize that spatial image transformations draw on a number of general-purpose spatial processing resources and a small number of transformation-specific resources. We refer to this view as the multiple systems framework (Zacks and Tversky, in press; Zacks, Mires, Tversky, and Hazeltine, 2002; Zacks, Ollinger, Sheridan, and Tversky, 2002; Zacks, Vettel, \& Michelon, 2003).

According to the multiple systems framework, imagined object-based transformations, imagined perspective transformations, and imagined effector-based transformations have much in common. In particular, each kind of transformation depends on representations in all three reference frames, which has implications for the interpretation of neuropsychological and neurophysiological data. For example, if one's objectbased reference frames are damaged, then performance of tasks requiring perspective transformations will be affected because perspective transformations update the relationship between egocentric and object-based reference frames. In general, a given task is likely to depend 
on representations in all three reference frames. Therefore, one cannot equate tasks with either representations or transformations.

The multiple systems framework also claims that a small number of neural-processing resources are transformation specific. For each transformation, we hypothesize that one or more unique neural structures mediate between representations in multiple reference frames to implement the updating of one of the reference frames. This process has two important implications. First, focal lesions may selectively impair one type of spatial transformation. Second, although different tasks requiring imagined spatial transformations would be expected to produce increased neural activity in a wide array of common processing resources, a small number of neural structures should be selectively activated by each transformation, and this activity should be modulated by the difficulty or extent of the transformation.

We hypothesize that the structures responsible for transformation-specific updating are shaped by natural selection and by learning within one's lifetime to capture regularities that are present in the environment (Shepard, 1994). One important type of regularity is that different situations are associated with different transformations. If imagined spatial transformations depend on the same processing machinery as actual transformations, then those regularities should be evident during spatial imagery and spatial reasoning. For example, body parts should be strongly associated with transformations of effector-centered reference frames. This hypothesis means that other things being equal, a spatial-reasoning task performed with pictures of hands or feet should be more likely to give rise to effector-based transformations than the same task using pictures of things other than body parts. In the same way, planning actions on small objects that one can manipulate should be strongly associated with transformations of object-centered reference frames. Thus, a spatial task involving small objects should be more likely to involve object-based transformations than the same task involving larger objects that would typically remain stationary in the environment.

Why would separate neural structures arise for different types of spatial reference frame updating? A more parsimonious alternative is that one general-purpose mechanism is responsible for computing any updating of the relations between spatial reference frames (Rock, Wheeler, and Tudor, 1989). We hypothesize that separate transformation-specific updating mechanisms arise for two reasons. First, implementing a general-purpose updating mechanism would likely be more expensive in terms of axonal connectivity between spatial representations. Second, a general-purpose updating mechanism would be less able to take advantage of features specific to each type of spatial transformation updating. For example, the object-based updating mechanism may be optimized for relatively small objects because large objects are less likely to undergo object-based transformations. The perspective updating mechanism may be optimized for transformations in the horizontal plane because horizontal movements are most common in experience and most important for action planning (at least in ground-dwelling species).

The account given here of the relationship between spatial reference frames and spatial transformations is a logical analysis, which we hope is helpful in thinking about visuospatial imagery whether or not one believes the multiple systems framework to be true. The multiple systems framework, however, is an empirical hypothesis, which is empirically falsifiable. In the following three sections we review research on object-based transformations, perspective transformations, and the interaction of effector-based transformations with visuospatial reasoning, which motivated the multiple systems view. We then describe studies that provide direct tests of its implications. Those data generally support the multiple systems framework; in particular, the data suggest that dissociable neural systems subserve object-based transformations and perspective transformations.

\section{OBJECT-BASED TRANSFORMATIONS}

\section{Behavioral Data}

Object-based transformations are the most studied visuospatial image transformations. Most of this attention has focused on one subclass of object-based spatial transformation: mental rotation. In mental rotation, the reference frame of an object is rotated relative to the egocentric and environmental reference frames. Mental rotation has been studied using two main types of tasks (see Figure 1). In comparison tasks, participants view two objects and are asked to decide whether they are identical, subject to rotation. The objects are usually either identical or mirror images, and from trial to trial the disparity in orientation between them is varied. The degree of rotation is usually hypothesized to be the length of the shortest rotational path between the two objects (Shepard and Metzler, 1971). In classification tasks, participants view a single object and are asked which of two versions of the object have been presented. The two possibilities are usually right-handed/lefthanded (for bodies or body parts) or normal/mirrorreversed (for alphanumeric characters). The orientation of the object is varied from trial to trial. In these tasks, the degree of rotation is usually hypothesized to be the disparity between the presented orientation and a canonical orientation determined by previous experience. For example, for alphanumeric characters the canonical orientation is assumed to be upright. A robust 
Comparison Task

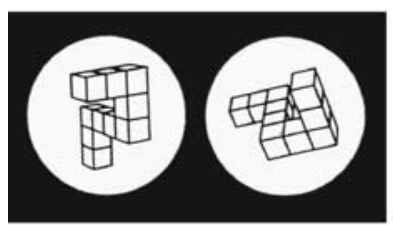

Are the two objects identical?

Figure 1: Examples of shape comparison and shape classification tasks.

SOURCE: Adapted from Shepard \& Metzler (1971) and Cooper \& Shepard (1973).

result obtained with both methods is that response times increase monotonically, and often linearly, as the degree of rotation increases (Shepard and Cooper, 1982).

Transformations in which an object's reference frame is translated, rather than rotated, relative to the egocentric and environmental reference frames also have been studied, though they have received somewhat less attention. One approach has used the mental-scanning task developed by Kosslyn and colleagues (Kosslyn, Ball, and Reiser, 1978). In this task, participants study a spatial representation, often a map, and then are explicitly instructed to imagine moving a mental marker from point to point in their mental image of the map. Across a number of studies, response times have increased with the distance of the path traversed, leading researchers to conclude that people perform the task by imagining that the reference frame of a mental marker is transformed so as to traverse the path (Denis and Kosslyn, 1999). Researchers also have used comparison tasks to study object-based translations. When the distance between two objects in the picture plane is varied and participants are asked to decide whether the objects are identical, response times increase with increasing distance (Larsen and Bundesen, 1998). A natural interpretation of this pattern is that the increasing response times reflect an object-based transformation in which the reference frame of one object is moved until aligned with that of the other. However, an alternative is that these effects reflect shifting of attention between the two depictions to compare features between them, with larger shifts taking longer. In shape-comparison tasks in which the two objects differ in size, it is generally found that response times increase monotonically with the ratio of the objects' sizes (Bundesen and Larsen, 1975; Larsen and Bundesen, 1978; Larsen, 1985). One possibility is that in these tasks, participants mentally translate one of the objects in depth until it is aligned with the other. Another possibility is that they may imagine that one object is scaled-a nonrigid deformation-until it is the same size as the other. Together, these results converge with the results from mental rotation experiments in suggesting that object-based transformations are implemented by continuously transforming the reference frame of an object relative to the other available reference frames. The fact that increases in response time are almost always monotonic and often linear suggests that the representational medium is an analog of threedimensional space and that the path taken is usually the shortest possible in this representational medium (Shepard and Cooper, 1982). (However, as will be seen in the section on effector-based transformations and motor imagery, in some cases, the paths taken are clearly not the shortest possible in Euclidean space.)

Not every possible object-based transformation can be readily simulated by every observer. Rather, there are strong limitations on people's ability to imagine object movements. Studies using shape-matching tasks indicate that imagined rotations are more difficult when they occur in planes that are oblique to the environmental or object-centered reference frames (Parsons, 1987b) particularly for those who are not of high spatial ability (Just and Carpenter, 1985). One interesting attribute of shape-matching tasks as they are usually conducted is that the test object depicts the post-transformation orientation of the to-be-transformed object. This depiction may be helpful in guiding the spatial transformation. When participants in one study were asked to imagine an object rotating around a specified axis and then place a second object in the orientation at which the rotated object would wind up, the difficulties observed with oblique axes became quite dramatic (Pani, 1993). When the plane of rotation was oblique with regard to both the object-centered and environmental reference frame, participants were essentially unable to do the task. These data suggest that people's object-based transformation abilities are optimized for simple rotations, where simple means rotations about the object's principal axis or the principal environmental axis.

\section{Neuropsychological Data}

Neuropsychological data can provide constraints on the neurophysiological substrate of object-based transformations. However, as noted by De Renzi (1982, chap. 6 ), the interpretation of these data requires some caution. Impairment on a particular spatial-reasoning task could result from damage to whatever structures are responsible for updating the relationship of objectbased reference frames to other references, but it also could result from a reduced ability to perceive the relevant spatial information or from an impairment of the object-based, egocentric, or environmental reference frames. Neuropsychological studies have mostly used 
shape-comparison tasks or construction tasks in which participants have to identify whether complex shapes can be constructed by assembling simpler shapes. For example, in the Minnesota Form Board test, participants are presented with a complex shape that can be constructed using a subset of five simpler shapes drawn below it, and they have to identify which of the simpler shapes to use (Likert and Quasha, 1948). Lesions to the posterior (particularly parietal) cortex lead to impairments on these tasks (De Renzi, 1982; Newcombe and Ratcliff, 1989). Farah (1989) reviewed a number of studies, focusing particularly on those using shapecomparison paradigms, and concluded that the data were conflicting: Across studies, deficits in mental rotation were associated with right posterior lesions, with right posterior or left anterior lesions, or with lesions to the posterior cortex in either hemisphere. One study found that direct electrical stimulation of the right parietal cortex selectively impaired mental rotation performance but did not affect performance of a control task that did not require performing a spatial transformation (Zacks, Gilliam et al., 2003). However, in this study the left hemisphere was not tested. In another study, transcranial magnetic stimulation was used to electrically stimulate either the right or left parietal cortex during mental rotation, and only right hemisphere stimulation led to impairments in performance (Harris and Miniussi, 2003). In sum, the neuropsychological data converge in indicating that the parietal cortex is important for performing object-based transformations, and the weight of the data suggests that the right hemisphere is more involved than the left. However, the question of hemispheric laterality is still a matter of some dispute.

\section{Functional Neuroimaging Data}

Converging evidence regarding the neural substrate of object-based transformations comes from functional neuroimaging. There have been a large number of neuroimaging studies of mental rotation, but, surprisingly, no comprehensive review of this literature has been published to date. To characterize the available data, we undertook a quantitative meta-analysis of the published studies. The full method and results will be reported elsewhere (Zacks, unpublished manuscript); here we provide a summary of the methods and conclusions.

We identified neuroimaging studies of mental rotation by searching abstract databases and, from those articles, transcribed reported foci of activation during performance of a mental rotation task. Task comparisons were classified as transformation-specific if they isolated within-task effects of mental rotation, for example, by comparing a condition involving rotated stimuli to a condition in which the stimuli were not rotated but the

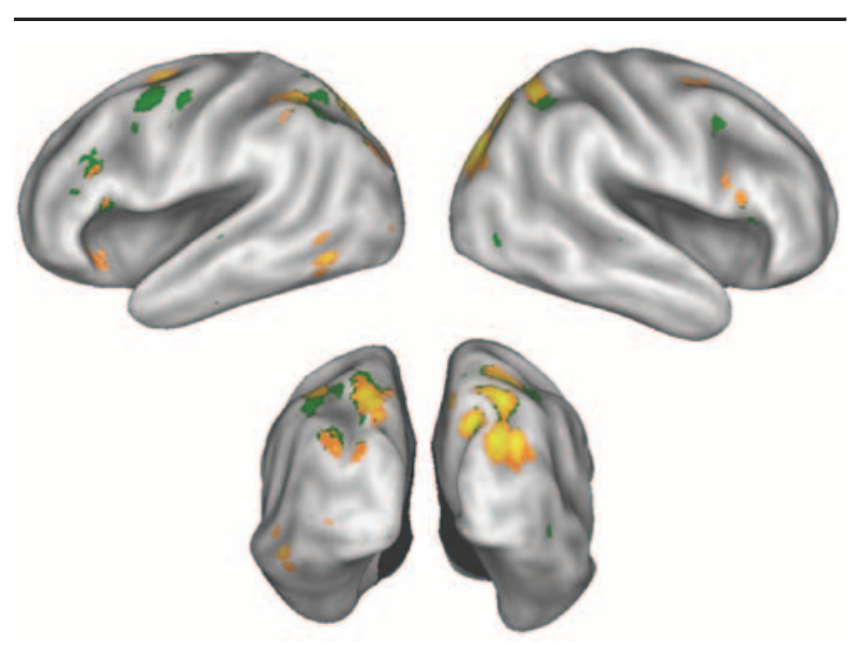

Figure 2: Meta-analysis of functional neuroimaging studies of mental rotation.

NOTE: Regions in orange-yellow were found to be reliably activated across studies in within-task comparisons that isolated mental rotation. Regions in green were associated with comparisons between mental rotation tasks and loose control states but were not reliably associated with mental rotation-specific contrasts. For both analyses, the voxelwise error rate was set at $p=.001$ (Turkeltaub et al., 2002). The upper images show lateral inflated views of the cortical hemispheres, and the bottom images show posterior inflated views. (All regions identified on the medial surface are visible in the posterior views.)

same task was performed. Task comparisons were classified as omnibus if they compared a mental rotation task to a loose control, for example, looking at a fixation crosshair. The reported foci were converted to probability maps, which were projected onto the cortical surface and visualized in Figure 2, with the transformation-specific map shown in orange-yellow overlaid on a more inclusive map, including both the omnibus and transformationspecific foci, shown in green. ${ }^{3}$

The most consistent activity associated with mental rotation was observed in the bilateral dorsal posterior cortex, extending from the lateral occipital cortex up through the posterior extent of the inferior parietal lobule, corresponding to Brodmann's areas (BA) 7 and 19. As can be seen in the figure, this association was stronger in the right hemisphere. This large mass of consistently activated brain tissue likely reflects several different neural-processing components. Part of the activity may reflect processes specific to updating objectcentered reference frames, but it is also likely that some of the observed activity reflects spatial and attentional processing related to the tracking of visual objects (Culham et al., 1998). Some of this activity may reflect more general cognitive processes such as focusing on the visual input or shifting spatial attention from one location to another (Corbetta and Shulman, 1998). 
Activity was also consistently observed in the precentral sulcus bilaterally, probably corresponding to the supplementary motor area and lateral premotor cortex (BA 6), and was more reliable in the left hemisphere. One possibility is that the activated regions correspond to the frontal eye fields, which are known to be involved in controlling overt eye movements and covert shifts of visual attention (Paus, 1996). Together with the occipital and parietal cortex, the frontal eye fields are often activated during visual object track and visual search tasks (Corbetta and Shulman, 1998; Culham et al., 1998). Another possibility is that they correspond to parts of lateral premotor cortex involved in the planning of hand movements (Picard and Strick, 2001), and possibly in the mental simulation of such movements (e.g., Deiber et al., 1998). We will return to the role of simulated movements in mental rotation in the section "Effector-Based Transformations and Motor Imagery" below.

Smaller areas of activity were observed in the left occipitotemporal sulcus (BA 19/37), the left inferior frontal gyrus (BA 45), and bilaterally in the ascending limb of the Sylvian fissure and the precentral sulcus (BA $44 / 45)$. The occipitotemporal activity may reflect processing related to the analysis of objects' shapes, which would be required for the tasks used in these studies. The prefrontal activity may reflect the working memory demands of these tasks (e.g., Awh et al., 1999), which likely increase with increasing stimulus orientation.

Including omnibus task contrasts in the analysis revealed additional regions in the bilateral lateral inferior premotor cortex (BA 6/9). These regions likely correspond to hand regions of the premotor cortex required for planning button-press responses often used in these tasks. This looser analysis also produced an increase in the anterior extent of the regions observed in the right parietal cortex and an increase in the extent of activity in the left lateral inferior parietal lobule. This last finding indicates that studies with tighter controls were more likely to report right-lateralized activity associated with mental rotation.

In short, the available neuroimaging studies of mental rotation provide strong evidence for the involvement of lateral occipital and parietal cortex in object-based transformations. The data suggest that the right hemisphere may play a more important role, but the studies included in this analysis were not specifically designed to test this hypothesis. These studies also suggest a role for the premotor cortex in mental rotation.

\section{PERSPECTIVE TRANSFORMATIONS}

\section{Behavioral Data}

Perspective transformations have received less attention in experimental psychology than object-based trans- formations, but a steady stream of data has accumulated. One line of research grew out of developmental psychology (for a review, see Newcombe, 1989), particularly Piaget's characterization of young children as having an egocentric bias. According to this view, the ability to imagine the world from a different viewpoint emerges relatively late in development, reaching adultlike competence at 9 or 10 years (Piaget and Inhelder, 1956). Until this stage, when children are asked to produce or identify visual depictions of another person's point of view, they tend to simply reproduce the primary features of their own view. To study this, Piaget and colleagues developed a task in which participants are shown a tabletop model of three mountains and a doll sitting at another position at the table and are asked to make judgments about how the scene looks to the doll. Several different judgment tasks have been used, including choosing the correct view from a set of pictures, constructing a model from the doll's view, or indicating whether the doll can see a particular object. Flavell and colleagues have distinguished between the sort of perspective taking that is required to answer whether another person can see a given object and the sort that is required to answer where an object is located relative to that person (Salatas and Flavell, 1976; Flavell, Everett, Croft, and Flavell, 1981). Recent data indicate that only the second sort of question requires perspective transformations (Michelon and Zacks, in press).

The previous section showed that most research on object-based transformations has focused on rotations. The same is basically true for perspective transformations, in part because much of the research has built on the Piagetian three-mountain task. However, whereas for object-based transformations the axis of rotation has almost always run through the object being rotated, for perspective transformations, the axis of rotation has often been outside of the person imagining the rotation. In this vein, a number of studies have used tasks in which participants are asked to look at an array of objects and imagine themselves rotating around the array; then they are asked to make a judgment about the array as viewed from the imagined perspective (Huttenlocher and Presson, 1973; Huttenlocher and Presson, 1979; Presson, 1980, 1982; Amorim and Stucchi, 1997; Creem, Wraga, and Proffitt, 2001). A smaller number of experiments have examined rotations in which participants are placed in the middle of an array of objects and asked to imagine themselves rotating in place (Wraga, Creem, and Proffitt, 2000; Carpenter and Proffitt, 2001; Creem, Downs, Wraga, Proffitt, and Downs, 2001; Wraga, Creem, and Proffitt, 2004; Wraga, Shepard, Church, Inati, and Kosslyn, 2005). In both cases, the transformation to be performed is conveyed to the participant either by visually indicating the location 
from which they should imagine viewing the scene or by verbally describing the direction and amount of movement. These two paradigms produce similar results. In both cases, response times and error rates are greater for trials requiring a transformation than for zero-rotation trials. Response times generally increase from $0^{\circ}$ to $180^{\circ}$, often as a negatively accelerated function. Beyond $180^{\circ}$, response times sometimes increase slightly with increasing rotation (e.g., Zacks, Vettel, et al., 2003) and sometimes decrease slightly (e.g., Wraga et al., 2000). Based on the observation that the largest response time increases typically are observed between $0^{\circ}$ and $90^{\circ}$, some researchers have proposed that viewer rotations may be performed as a "blink" transformation, in which a new visuospatial representation is generated from a structural description of the situation rather than by continuously transforming the spatial reference frames (Wraga et al., 2000). One problem with this interpretation is that further increases in response time are consistently observed between $90^{\circ}$ and $180^{\circ}$. This result is congruent with the proposal of the multiple systems framework that imagined perspective transformations are shaped by experience with actual perspective transformations.

Another research approach, which grew out of work on the updating of one's actual position during navigation, has investigated translational as well as rotational perspective transformations. In these studies, participants typically view an array of objects, close their eyes, and then move or imagine moving in the environment. After the actual or imagined transformations, the participants are asked to indicate the location of objects in the environment relative to their new actual or imagined egocentric reference frame. In one study, participants' updating of their actual position without visual input was highly accurate. Imagined perspective translations also were relatively easy: They were performed relatively quickly and led to accurate pointing judgments. However, imagined perspective rotations were quite difficult, leading to large systematic errors in pointing judgments (Rieser, 1989). When participants are asked to combine imagined perspective translations and rotations, it is the rotational component that appears to lead to large errors (Presson and Montello, 1994), and participants sometimes respond as if they performed only the translational component and failed to perform the imagined perspective rotation (Klatzky, Loomis, Beall, Chance, and Golledge, 1998). It has been argued that when people are asked to imagine rotating in their environment, they may sometimes simply generate a new mental image of the view from the new location based on their long-term memory, that is, a blink transformation (Wraga et al., 2000), which may sometimes be more efficient than transforming one perspective to another (Brockmole and Wang, 2003).

Most studies of perspective transformations have involved simple rotations and translations in the ground plane, whereas studies of object-based transformations have focused primarily on rotations in the coronal plane (orthogonal to the direction of gaze) but have included other planes of rotation. Carpenter and Proffitt (2001) studied perspective transformations in the ground plane, the coronal plane, and the sagittal plane (which separates the left and right sides of the body), using a procedure in which participants were instructed to imagine rotating a particular amount in a particular direction. Although Carpenter and Proffitt did not directly compare the three planes, in their experiments it appears that perspective transformations in the coronal and sagittal planes were more error prone than those in the ground plane. Creem, Wraga, and Proffitt (2001) found that perspective transformations were particularly difficult when they were oblique to the principal environmental axes. Parsons (1987a) studied perspective transformations involving rotations through these three planes and 10 oblique planes. He used a procedure in which participants were shown a picture of a body and asked to imagine themselves in the same position as the depicted figure. In this study as well, rotations in the ground plane led to the fastest response times. One possibility is that ground plane rotations are fast and fluent because people have more experience with them; another possibility is that in ground-dwelling species such as humans, ground plane transformations are subject to greater evolutionary selection.

\section{Neuropsychological Data}

The effects of brain damage on perspective transformations are poorly understood. One set of studies used a task in which participants had to walk a route marked on a simple map (Semmes, Weinstein, Ghent, and Teuber, $1955,1963)$. This task may depend especially on perspective transformations to keep one's actual heading aligned with the imagined heading represented on the map. Patients with left or bilateral posterior lesions (or left anterior lesions) were significantly impaired, whereas patients with right posterior lesions were not. However, Butters, Barton, and Brody (1970) found that both left and right parietal patients were impaired on a version of the Piagetian perspective-taking task described previously. The tasks used by these two groups differed substantially in their input and output modes and in their attentional requirements, which may have contributed to the lack of agreement.

Some hints about how perspective transformations might be implemented come from patients who have difficulty navigating in their environment. Such topographi- 
cal disorientation can arise as a result of lesions in a variety of brain areas and can reflect impairments of either egocentric or environmental reference frames (Farrell, 1996). In a recent synthesis of the literature, Aguirre and D'Esposito (1999) have proposed a distinction between two different types of spatial deficit relevant to navigation. ${ }^{4}$ In egocentric disorientation, patients are unable to represent the location of objects relative to themselves. This deficit is proposed to reflect damage to representations in egocentric reference frames and is associated with lesions to the posterior parietal cortex, particularly the superior parietal lobule and particularly the right hemisphere. In heading disorientation, patients are unable to maintain accurate representations of which direction they are facing relative to the environmental reference frame. This deficit is associated with lesions to the posterior cingulate cortex, particularly in the right hemisphere. (However, it is important to note that the total number of such patients is small.) Specific deficits in updating one's location during locomotion also have recently been identified in patients with lesions to the medial temporal lobe (Philbeck, Behrmann, Levy, Potolicchio, and Caputy, 2004). These patients generally can locate objects relative to themselves and relative to the reference frame of a room or house, so it would seem that both the egocentric and environmental reference frames are intact. Thus, such patients may have a selective deficit in updating their egocentric reference frame during locomotion, a failure that could involve the same mechanisms as those involved in imagined perspective transformations. It would be of great interest to test patients with heading disorientation on spatial updating and imagined perspective transformation tasks.

The available neuropsychological data suggest that the ability to perform tasks involving perspective transformations can be impaired by damage to the posterior parietal cortex, but this inability could result from damage to one or more spatial reference frames rather than from damage to transformation-specific processing resources. Cases of heading disorientation make the intriguing suggestion that the posterior cingulate cortex or medial temporal cortex may implement updating of the egocentric reference frame during locomotion and, therefore, possibly during imagination as well.

\section{Functional Neuroimaging Data}

There have been only a small number of neuroimaging studies of perspective transformations. The results of these are summarized in Figure 3. In one experiment (Zacks, Rypma, Gabrieli, Tversky, and Glover, 1999), participants viewed a picture of an upright man with one outstretched arm who faced either toward or away from the viewer. They were asked which of the man's arms was outstretched, and it was hypothe-
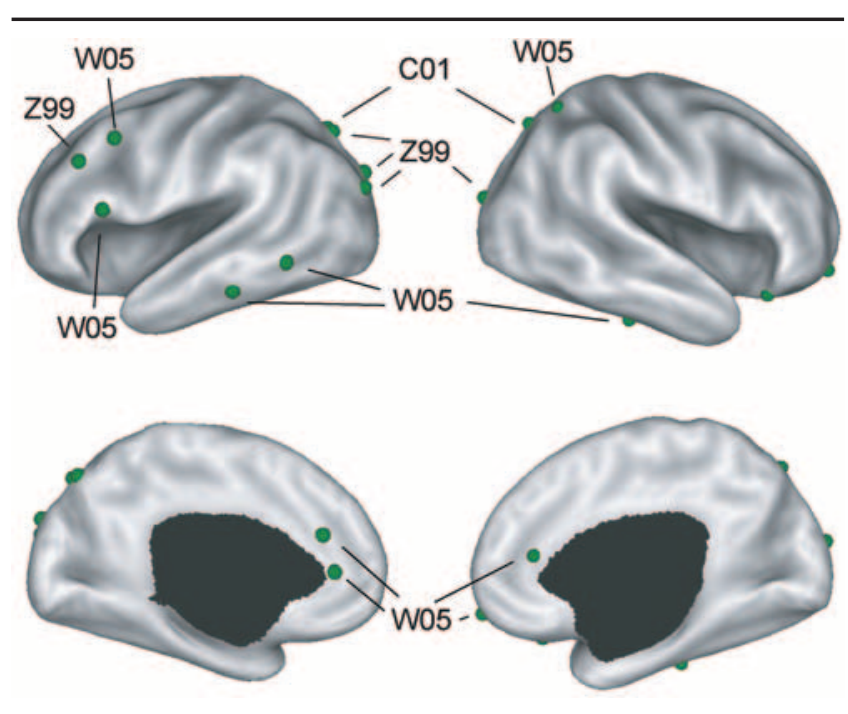

Figure 3: Summary of studies reporting selective increases during perspective transformations compared to a control condition.

NOTE: Labels indicate publication source. Foci that appear in both the lateral and medial views are labeled only in one of the two views. C01: Creem, S. H., Downs, T. H., Wraga, M., Proffitt, D. R., \& Downs, J. H., III. (2001). A functional magnetic resonance imaging (fMRI) study of imagined self-rotation. Cognitive, Affective $\mathcal{E}$ Behavioral Neuroscience, 1, 239-249. W05: Wraga, M., Shephard, J. M., Church, J. A., Inati, S., \& Kosslyn, S. M. (2005). Imagined rotations of self versus objects: an fMRI study. Neuropsychologia, 43, 1351-1361. Z99: Zacks, J. M., Rypma, B., Gabrieli, J., Tversky, B., \& Glover, G. (1999). Imagined transformations of bodies: an fMRI study. Neuropsychologia, 37, 1029-1040. (Stereotactic coordinates were not reported for Z99, so the locations of activation clusters greater than $1 \mathrm{cl}^{3}$ were estimated from the published figures.)

sized that they would solve this problem by imagining themselves in the position of the man. Compared to a control condition, this exercise led to activity in the posterior cuneus, the precuneus, the occipital and lingual gyri, and the superior parietal lobule, with much stronger activity in the left hemisphere than in the right hemisphere. Left-lateralized activity was also observed in the middle frontal gyrus, and bilateral activity was observed in the middle temporal gyrus. Two other studies used viewer rotation tasks, in which participants were asked to imagine moving and then make judgments about objects external to their bodies. In one experiment (Creem, Downs, et al., 2001), participants were placed supine in a functional magnetic resonance imaging scanner and viewed an array of objects in a pair of video goggles. They were asked to imagine lying in the middle of the array and rotating around their long axis (as if rolling on their sides) and then to report the location of objects relative to the imagined orientation. Compared to zero rotation control trials, this exercise led to increased activity in the precuneus, primarily in the left hemisphere. The second experiment examined rota- 
tional perspective transformations (as well as objectbased transformations) in oblique planes using a similar task (Wraga et al., 2005). Comparing perspective transformations to a no-rotation control condition led to activity in the prefrontal cortex, the right inferior frontal cortex, the medial frontal cortex, the bilateral fusiform gyrus, and the right superior parietal lobule. In sum, the neuroimaging data suggest that dorsal stream components of the posterior cortex, as well as the lateral frontal cortex, particularly in the left hemisphere, are important for perspective transformations. None of these studies provided evidence for activity in the posterior cingulate cortex or the medial temporal lobe during imagined perspective transformations, a finding seemingly at odds with the neuropsychological data on heading disorientation. One possibility is that heading disorientation reflects a disruption of the vestibular and kinesthetic inputs to the updating of egocentric reference frames. Such processing would be evident only for actual movement, not imagined perspective transformations.

\section{EFFECTOR-BASED TRANSFORMATIONS AND MOTOR IMAGERY}

When someone imagines moving, spatial representations in effector-centered reference frames are updated relative to the object-based and environmental reference frames. This type of updating is at the core of the motor simulations that occur when people imagine performing a body movement, that is, when they engage in motor imagery (Michelon et al., manuscript submitted for publication). (Henceforth, we will use the term motor imagery to describe tasks in which people are asked to imagine moving body parts and reserve the term effectorbased transformations for the process of updating effectorcentered reference frames.) Our treatment of such effector-based transformations will be brief, for two reasons. First and foremost, motor imagery is a well-studied phenomenon in its own right, and there are excellent review articles available (Jeannerod, 1995; Decety, 1996; Jeannerod and Frak, 1999). Second, although imagined body movements clearly are spatial transformations, they do not appear to be primarily visuospatial ones. Rather, the critical representations that are updated appear to capture motor and kinesthetic properties rather than visuospatial properties. Here, we will focus on the influence of effector-based transformations on the updating of visuospatial representations.

\section{Behavioral, Neuropsychological, and Functional Neuroimaging Data}

Just as visuospatial imagery shares mechanisms with visual perception, there is strong behavioral evidence that motor imagery and motor execution share common mechanisms. The time to move one's hand to a target decreases with the size of the target and increases with the distance to be moved, a relationship known as Fitts' law. This relationship holds for imagined as well as for actual movement (Jeannerod, 1995). Brain lesions that affect this relationship during performance have similar effects on tasks in which participants imagine moving (Sirigu et al., 1996; Sirigu and Duhamel, 2001). On a finer scale, the time to imagine moving one's hand into a particular position is highly correlated with the time it actually takes to perform that movement (Parsons, 1994). Imagining moving one's limb leads to subtle but detectable changes in the peripheral musculature, which can affect reflex sensitivity and, in some cases, may lead to electrical activity in the muscles (Jeannerod, 1995). In sum, the behavioral, neurological, and psychophysiological data suggest that when participants are asked to imagine performing a movement, they draw on mechanisms that overlap significantly with the mechanisms that control voluntary motor action.

What are the neurophysiological substrates of these mechanisms? This question can best be addressed in terms of the hierarchical organization of the primate motor system (Dum and Strick, 2002). The primary motor cortex (M1) projects to the spinal cord and shows strong somatotopic organization, with nearby areas of the body being represented by nearby cells. Stimulation of M1 produces simple movements at relatively low thresholds. A set of areas collectively called the premotor cortex lie anterior to the M1 and project to it (as well as projecting directly to the spinal cord). The more posterior premotor regions, including the supplementary motor area and the ventral premotor cortex, evidence moderately strong somatotopic organization and appear to be more directly tied to motor execution, whereas the more anterior regions show weak (if any) somatotopic organization and may play roles in target selection, attention, or sequencing. Another set of regions involved in motor control, also with spinal projections, lies in the middle of the cingulate gyrus. These regions also appear to have a posterior-to-anterior organization, with the more posterior regions more directly involved in motor control.

Activity in these areas during imagined movements has been studied with positron emission tomography (PET) and functional magnetic resonance imaging (fMRI) (for reviews, see Jeannerod and Frak, 1999; Grézes and Decety, 2001; Decety, 2002). Most of the studies used tasks in which participants performed sequential finger movements or imagined performing such actions. These paradigms give rise to robust activation in posterior premotor regions during imagined movements. In 
some studies, activity has also been observed in the M1, though the evidence for this activity is weaker.

\section{Interactions Between Motor Imagery and Object-Based Transformations}

Stimulation of the M1 and the premotor cortex generally does not give rise to visuospatial percepts (Penfield and Rasmussen, 1950). This fact and the data just reviewed suggest that the effector-based transformations primarily involve kinesthetic and somatosensory representations rather than visuospatial ones. However, there is converging evidence that these nonvisual representations interact with visuospatial representations during spatial imagery and spatial reasoning-particularly object-based transformations. One source of evidence comes from behavioral studies. In classification and comparison tasks used to study mental rotation, one finds apparent effects of motor imagery on response times. The time to classify pictures of hands as left or right generally increases with increasing rotation from upright, as for other objects. However, there are robust deviations from monotonicity, and these deviations vary with the hand being tested (Cooper and Shepard, 1975; Sekiyama, 1982; Parsons, 1994; Parsons, Gabrieli, Phelps, and Gazzaniga, 1998; Thayer, Johnson, Corballis, and Hamm, 2001; Wohlschläger, 2001). Moreover, as noted previously, there is a strong correlation between the time to classify a picture of a hand as left or right and the time to move one's hand into the position shown in the picture (Parsons, 1994). These results suggest that people classify hands as left or right by mentally simulating a movement of their own hand. The effects of motor processing on mental rotation have been observed with other stimuli as well. In three studies, when participants were asked to physically rotate their hands while performing shape-comparison tasks with geometric objects, response times were slowed when the physical and mental rotation directions conflicted and were speeded when the physical and mental rotation directions matched (Wexler, Kosslyn, and Berthoz, 1998; Wohlschläger and Wohlschläger, 1998; Schwartz and Holton, 2000).

A second source of evidence that object-based transformations interact with effector-based transformations comes from neuropsychological and neuroimaging studies. In one study, pictures of left or right hands were presented selectively to the left or right hemispheres of patients who had undergone callosotomies (severing of the corpus callosum) to treat intractable epilepsy. These patients were severely impaired at identifying left hands presented to the left hemisphere and right hands presented to the right hemisphere, suggesting that correct performance depended on a motor representation in the hemisphere contralateral to the depicted hand (Par- sons et al., 1998). Consistent with this result, a neuroimaging study of hand classification found contralaterally organized activity in the premotor cortex of neurologically healthy adults during hand classification (Parsons et al., 1995). Neuroimaging studies of shape-comparison tasks also have provided evidence for interaction between effector-based transformations and object-based transformations. A number of studies have reported activity in motor regions, particularly in the left hemisphere, during mental rotation tasks (see "ObjectBased Transformations" above and also Vingerhoets, de Lange, Vandemaele, Deblaere, and Achten, 2002 for reviews). Two studies have linked the magnitude of activity in motor areas to response time (Richter, Ugurbil, Georgopoulos, and Kim, 1997; Tagaris et al., 1997). However, these activations may reflect activity in anterior premotor areas that are not directly involved in motor control or motor simulation and, in some cases, may reflect activity related to the button-press responses often required in these tasks. Stronger evidence comes from four studies that varied the putative contribution of motor imagery to a shape-comparison task. One study contrasted shape-comparison judgments about pairs of geometric stimuli with shape-comparison judgments about pairs of hands (Kosslyn, DiGirolamo, Thompson, \& Alpert, 1998). Judgments about hands led to greater activity in the M1. A similar study compared judgments about hands to judgments about tools (Vingerhoets et al., 2002). Judgments about hands led to significant bilateral increases in the premotor cortex, whereas judgments about tools produced significant increases only in the left premotor cortex. However, in a direct comparison, no areas showed significantly greater activation for mental rotation of hands than tools. In the third study, participants were trained either to passively view geometric objects rotating or to manually rotate them, and then they performed a shape-comparison task with geometric objects during PET scanning while refraining from moving (Kosslyn, Thompson, Wraga, and Alpert, 2001). Only the group previously trained to rotate the objects showed increased activity in the M1 and the premotor cortex, suggesting that they transferred a motor strategy from the initial training. The fourth study also used a training transfer paradigm (Wraga, Thompson, Alpert, and Kosslyn, 2003). In this experiment, one group of participants initially performed a shape-comparison task with pictures of abstract geometric objects, and the other group performed a motor-imagery task (a shapecomparison task with pictures of hands). Both groups then performed the shape-comparison task with abstract objects. PET recording found that brain activity increased in the M1 and the premotor cortex only in the group that had initially performed motor imagery, suggesting that the participants in that group had trans- 
ferred a strategy including effector-based transformations from the first task. In sum, the behavioral and neurophysiological data suggest that effector-based transformations may interact with object-based transformations when the stimuli or task demands encourage it. The available data do not provide strong evidence that object-based transformations obligatorily require the updating of effector-centered representations, but this possibility cannot be ruled out at this time.

\section{Interactions Between Motor Imagery and Perspective Transformations}

If effector-based transformations interact with objectbased transformations, one might also expect them to interact with perspective transformations. Unfortunately, there are very few data that bear on this hypothesis. The closest relevant data are those of Parsons (1987a), described previously. In that study, participants were shown pictures of human bodies and asked either to imagine themselves in the position of the depicted body or to answer whether the outstretched arm of the depicted body was a left arm or a right arm. The two tasks led to highly similar systematic relationships between stimulus orientation and response time. Moreover, these relationships deviated strongly from the monotonic increases that would be expected for an object-based transformation. Based on these findings, Parsons argued that participants in both tasks performed motor simulations to place themselves in the depicted position. This possibility cannot be ruled out, but to provide strong evidence for this interpretation would require asking participants to actually place themselves in each of the depicted positions and measure the time it took. However, most of the tested positions are physically impossible for most people. This fact in and of itself renders less plausible the possibility that participants performed detailed motor simulations in this case.

Research on spatial updating also provides suggestive-but inconclusive-evidence that systems for motor control may interact with systems that update one's egocentric reference frame. If people are blindfolded and then walked to a new location, they are often quite good at pointing to the locations of objects (e.g., Presson and Montello, 1994). However, if they are asked to imagine moving to that location, performance can be quite poor, as noted previously (see "Perspective Transformations" above). These data do not provide strong evidence for interaction between perspective transformations and effector-based transformations because the advantage incurred by motion could be attributed to vestibular cues to whole-body movement rather than to processing related to movements of body parts. Consistent with this possibility, passive blindfolded movement also leads to accurate pointing (Rieser, 1989; Sholl and Bartels,
2002). Stronger evidence for an influence of action planning, if not body movements as such, comes from a study in which people were rotated in a motorized apparatus while blindfolded (Féry, Magnac, and Isrâll, 2004). Rotations that participants could control with a joystick led to better updating than passive rotations.

Neuroimaging data could help illuminate the relationship between effector-based transformations and perspective transformations, but, again, here the data are thin on the ground. It is suggestive that in the neuroimaging studies reviewed in the previous section, no evidence has been found for the activation of the M1, the supplementary motor area, or the premotor cortex during perspective transformations. This finding stands in contrast to the reports of activity in the M1 and the premotor cortex during object-based transformations in some cases (see "Object-Based Transformations" above). Studies aimed at directly investigating this apparent difference would be valuable.

To summarize this section: Movement is controlled hierarchically, with the regions in the posterior frontal cortex playing a critical role in the mapping of high-level plans or intentions to sequences of muscle contractions. A significant component of these operations is shared between motor performance and motor imagery, and this overlap may include the processes responsible for updating effector-centered reference frames. Such motor processes likely interact with the processes that transform visuospatial representations during objectbased transformations, but it is not clear whether this is an obligatory concomitant of object-based transformations. Motor processes may also interact with the processes that transform visuospatial representations during perspective transformations, but there are few data on this point.

\section{DISSOCIATIONS BETWEEN OBJECT-BASED TRANSFORMATIONS AND PERSPECTIVE TRANSFORMATIONS}

The multiple systems view proposes that object-based transformations and perspective transformations share many common processing components but that each also depends on unique processing resources. The sharing of processing components implies that tasks requiring object-based or perspective transformations should share common behavioral and neurophysiological characteristics. As we have seen in the previous two sections, for tasks based on both types of transformation, response time can be affected by the angular or linear distance of the imagined transformation. We also saw that tasks based on object-based or perspective transformations both appear to depend on cortical regions including the posterior parietal cortex and adjacent areas in the occip- 
ital and temporal lobes. The multiple systems view also claims that object-based transformations and perspective transformations differ in a small number of processing components-those that implement the selective updating of a spatial reference frame relative to other reference frames. This view implies that under controlled circumstances, it should be possible to dissociate object-based and perspective transformations in terms of their behavior and neurophysiology. This section reviews studies of behavioral performance, individual differences, and neurophysiology that provide evidence for such dissociations. (See also Wraga et al., 1999, for a review of some of the relevant behavioral data.)

\section{Behavioral Data}

Several studies have used variants of the Piagetian three-mountain problem, described previously, to compare error rates and response times for object-based and perspective transformations. Huttenlocher and Presson (1973) designed a version in which children (grades 3 and 5) were asked to make judgments about the appearance of an array of objects if either the array were to be rotated (an object-based transformation) or if the children were in the position of an observer at another location (a perspective transformation) by choosing one picture from several pictures. The children made more errors on the perspective transformation task. A second experiment (with fourth graders) suggested that part of the poorer performance with self-rotations might be attributed to difficulty taking another's point of view because if the children were asked to imagine themselves moving rather than to imagine taking the point of view of another person, they performed better. A followup study compared the picture-recognition task to a question about the location of one of the objects in the array (Huttenlocher and Presson, 1973). As before, when third graders were asked to choose a picture corresponding to the appearance of the transformed array, self-rotations were easier than array rotations. However, when they were asked to identify the location of an object in the transformed array, rotations of the array were easier than self-rotations. The authors suggested that to perform the picture-recognition task, the children had to update the object-centered reference frame of each object in the array relative to the environmental reference frame. However, to perform the location-judgment task, they had only to update the reference frame of one object, making the array rotation condition easier. Another related study with college-aged participants again varied the judgment task that participants performed (Presson, 1982). The results were consistent with the view that participants update the reference frame of a single object rather than updating the array as a whole when possible. When participants were asked which item was in a given location, self-rotations were faster and more accurate than array rotations. However, when they were asked the location of a particular item or asked to construct a copy of the transformed array, array rotations were easier. Array rotations also have been found to be easier when the stimuli were simple symmetric line drawings (van Lier, 2003).

Recently, a comprehensive study of array and viewer rotations manipulated the location of the viewer relative to the array (inside or outside), the judgment question asked, and the ease with which one might code the array of objects as a single object with a single reference frame (Wraga et al., 2000). Again, array rotation problems were easier when participants were asked to name the location of a particular object than when they were asked which object was at a particular location. One experiment provided strong support for the notion that when possible, participants solve array rotation problems by updating only one of the objects. In this experiment, participants were usually asked to perform an imagined transformation and then indicate the location of a particular object. However, on a few trials, they were subsequently asked which object was in a particular location. If they had answered the first question by updating the entire array, performance on the second question should have been fast and accurate, but for array rotations this was not the case. Another experiment attempted to make it easier for participants to code the objects with a single reference frame by replacing the array with a block with sides of different colors or with a car that included multiple parts (door, headlights, etc.). Changing the array to a block did not reliably improve the ease of array rotations relative to viewer rotations, but changing the array to a car helped performance in the array rotation condition. In this study, viewer rotations were faster and more accurate than array rotations in all conditions.

This methodology has also been used to study imagined translations (Creem-Regehr, 2003). In this study, participants stood next to a long table with objects placed on it and were asked to imagine moving along the table or to imagine the table moving next to them, by a specified distance. They then were asked to name the color of the object that would be immediately beside them. In some conditions, the imagined movements were forward or backward; in others, they were left or right. In both cases, viewer translations were performed faster and more accurately than array translations.

These studies suggest that perspective transformations obligatorily affect the relationship between the egocentric reference frame and the reference frames of each object, but object-based transformations may be applied to individual objects, selectively updating that object's relationship to the egocentric reference frame, 
and this method appears to be the preferred strategy. As a result, object-based and perspective transformations differ in their effectiveness in supporting different spatial judgments (see also Newcombe, 1989, 2002). Data from actual viewer and array rotations suggest that updating the position of an array of objects as a whole is quite difficult (Simons and Wang, 1998; Wang and Simons, 1999). In these experiments, participants viewed an array of objects, which was then hidden from view. Either the participant or the array was rotated, and the array was uncovered. On some trials, objects were moved. Participants were very good at detecting these changes after they themselves were moved but poor at detecting changes after the array was moved.

As well as supporting different types of responding, object-based transformations and perspective transformations differ in their timing. In both of the imagined rotation studies that measured response times during array rotation and viewer rotation tasks (Presson, 1980; Wraga et al., 2000), response times in the array rotation condition increased monotonically with an increasing angle of rotation. However, response times in the viewer rotation condition were flatter overall and tended not to increase from $180^{\circ}$ to $270^{\circ}$. Manipulating the spatial judgment required after the transformation did not appear to affect this pattern. Similarly, in the study of array and viewer translation (Creem-Regehr, 2003), response times increased with increasing distance for array translations only.

The studies based on the Piagetian task depend on direct instruction to evoke object-based or perspective transformations. This technique has the advantages of face validity and transparency but depends on participants' ability to willfully call up the desired spatial image transformation and not "cheat." In particular, for $270^{\circ}$ rotations, participants may find it easier to violate the instructions and rotate themselves or the array $90^{\circ}$ in the direction opposite the one instructed (Wraga et al., 2000). Recent studies have used a converging approach, in which the experimenters vary the stimuli presented and the spatial judgment required to manipulate which type of transformation is performed. These studies have capitalized on an intriguing difference between response time patterns for object-based and perspective transformations (for a more thorough discussion, see Zacks and Tversky, in press). As noted previously, response times for object-based transformations consistently increase monotonically with an increasing angle of rotation (see "Object-Based Transformations" above). This outcome is thought to reflect the fact that the constraints on object-based transformations are isotropicthat is, the "cost" of rotation in any direction is equivalent-just as for physical rotations in space (Shepard and Metzler, 1971). However, this is not necessarily the case for perspective transformations; in fact, previous data indicate strong departures from monotonic increases in some planes of rotation. In particular, for some planes of rotation, it appears that perspective transformations may be effectively independent of rotational distance (Parsons, 1987a, see "Effector-Based Transformations and Motor Imagery" above).

In an initial experiment capitalizing on these response time differences (Zacks, Mires, et al., 2002), participants answered questions about line drawings of bodies. Two types of question were used (see Figure 4): For same-different questions, participants were shown two pictures of bodies with one outstretched arm, one above the other, with the top one always upright and the bottom one varying in orientation. They were asked whether the pictures were identical or mirror images (a comparison task). For left-right questions, participants were shown only the varying-orientation picture and asked whether the outstretched arm was left or right (a classification task). For both questions, the pictures were always drawn facing the viewer. It was hypothesized that to answer the same-different questions, participants would perform an object-based transformation to rotate the reference frame of the bottom picture to upright and that this strategy would lead to monotonic increases in response time. This hypothesis was based on the robust finding, reviewed previously, that in many situations, mental rotation is used to solve this sort of shapecomparison task. For left-right questions, it was hypothesized that participants would perform a perspective transformation, aligning their imagined egocentric reference with that of the picture and identifying the location of the outstretched arm relative to their transformed egocentric reference frame. This hypothesis was based on previous data showing that the time to make left-right judgments about pictures of bodies is strongly correlated with the time to imagine one's self in the position of a pictured body-and, for this particular plane of rotation, are independent of stimulus orientation (Parsons, 1987a). Thus, it was predicted that varying the spatial judgment would affect response time patterns, leading to monotonic increases in response time, with increasing rotation for the same-different task but not for the left-right task. This behavior is what was observed.

Introspective reports converge in indicating that people use object-based transformations to answer samedifferent questions about pictures of bodies but use perspective transformations to answer left-right questions about the same pictures (Zacks and Tversky, in press). In one experiment, participants performed one samedifferent or left-right trial and then were asked to describe how they solved the problem. All participants who made same-different judgments about bodies reported performing an object-based transformation, whereas $76 \%$ 


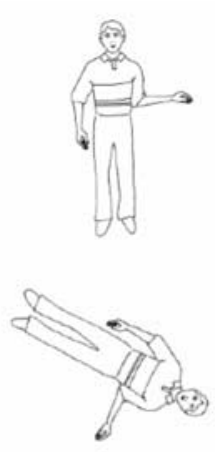

Are the two figures identical? (Answer: yes)

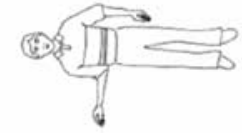

Is the figure's left or right arm extended? (Answer: right)

Figure 4: Examples of the same-different and left-right tasks. SOURCE: Adapted from Zacks, Mires, et al., 2002.

of participants who made left-right judgments reported performing a perspective transformation. Further evidence came from a second experiment using an interference paradigm: When participants were asked to answer same-different questions by performing perspective transformations or to answer left-right questions by performing object-based transformations, performance was impaired. However, asking participants to answer leftright questions by performing perspective transformations or to answer same-different questions by performing object-based transformations had no effect on response time or accuracy.

One claim of the multiple systems view is that neural systems for object-based and perspective transformations evolved to solve different sorts of problems. This claim implies that object-based transformations are tuned to planning actions on objects that one could physically manipulate, whereas perspective transformations are tuned to planning possible self-movements or social interactions. Thus, whether a spatial-reasoning task will evoke object-based or perspective transformations should depend not just on the type of question to be answered but also on the type of thing about which the question is asked. On this view, pictures of small objects should preferentially evoke object-based transformations because they are potential targets of physical manipulation. Pictures of large spaces should preferentially evoke perspective transformations because they are markers of potential perspectives one might take on an environment. Bodies have a special status because they function both as objects that we see being moved or moving themselves and as markers of potential perspectives on the world. Both response time patterns and introspective reports provide support for the hypotheses that pictures of small objects preferentially evoke object-

based transformations, and pictures of large spaces preferentially evoke perspective transformations. For both left-right and same-different judgments, pictures of small objects, such as drills and cups, lead to strong increases in response time with increasing rotation in the picture plane and to self-reports of performing object-based transformations (Zacks and Tversky, in press). Pictures of rooms led to relatively weak increases in response time with increasing picture plane rotation for same-different judgments as well as for left-right judgments (Zacks and Shelton, 2003).

\section{Individual Differences}

Individuals differ markedly in their ability to perform spatial-reasoning tasks. The multiple systems view predicts that across individuals, performance on tasks requiring object-based transformations should be correlated with performance on tasks requiring perspective transformations. This rather mundane prediction results from the claim that the two types of tasks share many computational resources, including visuospatial attention, memory, encoding, and readout. However, the multiple systems view also makes the riskier prediction that above and beyond this common variation across individuals, there should be unique individual differences associated with tasks depending on objectbased transformations and different unique individual differences associated with tasks depending on perspective transformations because the two sets of tasks depend on different types of reference frame updating. In a series of psychometric studies, Hegarty, Kozhevnikov, and Waller have recently provided evidence that is consistent with both of these predictions (Hegarty and Kozhevnikov, 1999; Kozhevnikov and Hegarty, 2001; Hegarty and Waller, 2004). In one study (Kozhevnikov and Hegarty, 2001), participants completed a battery of six spatial-reasoning tests, of which three were hypothesized to depend heavily on object-based transformations and two were hypothesized to depend on perspective transformations. The former included classic tests of mental rotation and paper folding. The latter were two versions of a novel test in which participants were shown a map with a number of objects on it and asked to imagine themselves standing at one object facing another and then to indicate the direction of a third object from their imagined perspective. (The final test could not be classified a priori as depending on object-based or perspective transformations.) Confirmatory factor analysis indicated that the correlations between scores on the various tests could not be accounted for well by positing a single spatial ability factor but were well fit by positing two factors, with the object-based tests loading heavily on one factor and the perspective-taking tests loading heavily on the other factor. In a second study (Hegarty and Waller, 
2004), two groups of participants completed batteries of spatial-reasoning tests that included tests thought to depend on object-based transformations or perspective transformations. The putative object-based transformation tests were classic mental rotation tasks, and the putative perspective transformation tests included versions of the new perspective-taking test, a test of map navigation, and a picture-matching test similar to the Piagetian tasks described previously. In both samples, factor analysis indicated that two spatial factors corresponding to object-based and perspective transformations fit the data significantly better than a single spatial ability factor. In sum, the available data on individual differences in spatial abilities support the claim that object-based transformations and perspective transformations depend on different computational systems, which vary in efficiency somewhat independently across individuals. It should be noted, however, that the library of tests of object-based transformations is much larger than the library of tests of perspective transformations. One of the important contributions of this group's research has been the development and validation of new tests of perspective taking.

\section{Neuroimaging Data}

Perhaps the most direct way to test the multiple systems view is to test for neurophysiological dissociations between object-based transformations and perspective transformations. Only three neuroimaging studies have directly compared object-based transformations and perspective transformations; these are summarized in Figure 5. The first used the left-right and same-different tasks described previously in an event-related fMRI design (Zacks, Ollinger, et al., 2002). Participants viewed pictures of human bodies and made left-right or samedifferent judgments about them. The orientation of the stimulus varied from trial to trial. A large volume of the brain showed task-related increases in activity, including the occipital, temporal, parietal, and posterior frontal cortex, and a substantial portion of the cerebellum. This result is not surprising and is consistent with the hypothesis that tasks requiring either object-based or perspective transformations share a large number of computational components. In addition, a small volume of brain showed larger task-related increases during the samedifferent task than during the left-right task. This brain activation was restricted to the right inferior occipital and temporal cortex and to the superior cerebellum bilaterally. No brain areas were found to be selectively activated during the left-right task.

The second study used a version of the viewer rotation and array rotation tasks described previously and, again, used an event-related fMRI design (Zacks, Vettel et al., 2003). Participants viewed a picture of a square board

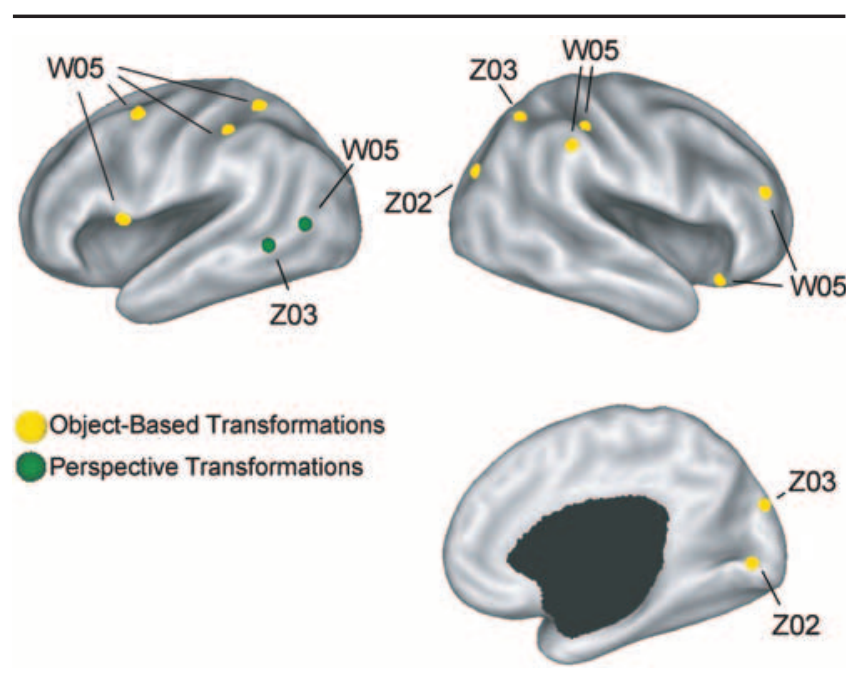

$\overline{\text { Figure } 5 \text { Summary of functional magnetic resonance imaging studies }}$ directly comparing object-based transformations and perspective transformations.

NOTE: Foci of selective increases in activity for object-based transformations are plotted in yellow, and selective increases for perspective transformations are plotted in green. Labels indicate publication source. In the Zacks et al., 2002, study, there was also a selective increase during object-based transformations in the medial superior cerebellum, which is not shown in the figure.

W05: Wraga, M., Shephard, J. M., Church, J. A., Inati, S., \& Kosslyn, S. M. (2005). Imagined rotations of self versus objects: an fMRI study. Neuropsychologia, 43, 1351-1361. Z02: Zacks, J. M., Ollinger, J. M., Sheridan, M. A., \& Tversky, B. (2002). A parametric study of mental spatial transformations of bodies. NeuroImage, 16, 857-872. Z03: Zacks, J. M., Vettel, J. M., \& Michelon, P. (2003). Imagined viewer and object rotations dissociated with event-related fMRI. Journal of Cognitive Neuroscience, $15,1002-1018$.

with four colored cubes mounted on sticks at the corners of the board. They were asked to imagine themselves rotating around the board by a specified amount (a perspective transformation) or to imagine the board rotating by a specified amount (an object-based transformation) and then answer a question about the imagined situation. In one experiment, the question was whether a particular cube would be on their left or right. In the other experiment, they were asked to identify the color of the cube that would be immediately in front of them on the left or right. Again, a large common volume of brain tissue was activated by performing these tasks. However, only two regions were selectively activated by one of the two tasks. One region, the left parietaltemporal-occipital junction, increased in activity significantly for perspective transformations but was largely inactive during object-based transformations. Another region, the right intraparietal sulcus, increased in activity for both tasks but increased significantly more during object-based transformations.

The third study also used a version of the viewer rotation and array rotation tasks in a block-design fMRI 
experiment (Wraga et al., 2005). Participants were shown a picture of an abstract geometric object in a sphere and a letter $T$ outside the sphere. In the objectbased transformation condition, they were asked to imagine the object rotating until a specified part was lined up with the letter $T$. In the perspective transformation condition, they were asked to imagine themselves in the position of the $T$. In both cases, they were then asked to indicate whether a specified part of the object would be visible. During task performance, large volumes of cortex were activated for both object-based transformations and perspective transformations. A single region in the left posterior cortex was selectively activated during the perspective transformation condition, in a location quite near that reported in the previously described study. A number of regions in the parietal, occipital, and frontal cortex that were selectively activated during the object-based transformation condition were reported.

This large number of regions specific to object-based transformations in the third study differs from the pattern observed in the first two studies. This finding may reflect a methodological difference between the experiments. In all three studies, the object-based transformation task was more difficult than the perspective transformation task, as indexed by response time and error rate. In the first two studies, these differences were controlled statistically in an event-related design, whereas in the third study, overall activity in the two tasks was compared using a block design. Thus, the additional areas observed to be selectively activated during object-based transformations in the third study may reflect processing that was common to both tasks, such as attention or memory, but that was engaged for a greater proportion of the task block during the task that was performed more slowly.

Thus, the limited available neuroimaging data converge in identifying a large number of brain regions that are commonly activated during tasks requiring objectbased and perspective transformations. A much smaller number of brain regions appears to be selectively activated by one or the other transformation. These regions are good candidates for implementing the selective updating of an object-based or egocentric reference frame. Regions that may be responsible for updating object-based reference frames include the right posterior parietal, occipital, and superior temporal cortex, as well as the superior cerebellum. The left parietaltemporal-occipital junction may be responsible for selective updating of egocentric reference frames.

In sum, the available behavioral and neuroimaging data support the multiple systems view. Although objectbased transformations and perspective transformations appear to share many computational and neural resources, they also differ consistently in their chronometry, psychometric properties, and neural correlates. These differences suggest that the computational mechanisms by which object-based reference frames and egocentric reference frames are selectively updated can be dissociated.

\section{DIRECTIONS FOR RESEARCH}

The research reviewed here establishes several key points. First, for each type of visuospatial transformation, there is a robust correspondence between imagined transformations and corresponding actual transformations. Second, visuospatial image transformations depend heavily on the posterior parietal, occipital, and temporal cortex. Third, unique processing resources play a role in each transformation type. Fourth, in common spatial-reasoning tasks, multiple reference frame updating systems may cooperate to facilitate task performance-at least in the case of object-based transformations and effector-based transformations. However, there is still a great deal to be learned about the specific roles of the neural systems identified here in visuospatial transformations and about the interactions between spatial updating systems during spatial reasoning. We think that the analysis presented here can be helpful in guiding future research, and in the following paragraphs, we will identify what we feel to be the most urgent outstanding problems.

Computational models of mental spatial image transformations are sorely needed. A small number of models of object-based transformations have been proposed (Funt, 1983; Just and Carpenter, 1985; Pinker, 1988), but this effort has languished in recent years. We believe the time is ripe to return to this problem with current computational techniques and neurophysiological data in hand. To our knowledge, there have been no computational models developed to account for perspective transformations. Formal models covering object-based transformations, perspective transformations, and effector-based transformations would be of singular value in guiding experimental and neuropsychological investigations-particularly for generating predictions that discriminate the different transformations quantitatively.

One important issue that such models should address-which we have skipped over here-is the fact that multiple conflicting spatial representations may coexist in the mind of an observer. In particular, representations of imagined states of affairs may compete with perceptually grounded representations. This factor seems to be particularly true for tasks in which locations must be reported in an imagined egocentric reference frame. Newcombe and Huttenlocher (1992) argue that such reference frame conflicts account for some of the 
classical errors in children's spatial reasoning. Recent studies suggest that some of the difficulty with rotational perspective transformations may reflect conflict between the location of objects in the actual and imagined egocentric reference frames (Farrell and Robertson, 1998; Wraga, 2003; Avraamides, Klatzky, Loomis, and Golledge, 2004). It seems that representations of the current egocentric location of objects exert a strong influence on some response measures, which should be systematically investigated and included in a comprehensive model.

Although object-based transformations, particularly mental rotation, have received a good deal of empirical attention, the other forms of mental image updating reviewed here are less well characterized. More behavioral, neuropsychological, and neurophysiological data on translational object-based transformations and on rotational and translational perspective transformations would be extremely helpful.

The data on individual differences reviewed in the previous section demonstrate the value of this approach to understanding the relationship between object-based transformations and perspective transformations. It would be beneficial to extend this approach to other task paradigms. It would also be valuable to apply this approach to the study of group differences. There is substantial and contentious literature on gender differences in mental imagery, which has focused largely on mental rotation (Geary, 1998; Kimura, 1999). Extending these investigations to include perspective transformations and effector-based transformations may help clarify some of the issues (Zacks, Mires, et al., 2002; Wraga et al., 2004). Along with gender differences, it would be valuable to extend these approaches to the study of age differences in spatial reasoning. We have seen in the previous sections that the development of perspective transformations during childhood has received some empirical attention, as has the development of mental rotation (Piaget and Inhelder, 1956; Kail, Pellegrino, and Carter, 1980; Kail, 1985; Newcombe, 1989). There have also been some studies of changes in visuospatial imagery throughout adulthood and aging. Most studies suggest that both object-based transformations and perspective transformations are affected by age (Flicker, Ferris, Crook, Reisberg, and Bartus, 1988; Aubrey and Dobbs, 1990; Hale, Lima, and Myerson, 1991; Hertzog, Vernon, and Rypma, 1993; Dror and Kosslyn, 1994; Hale, Myerson, Faust, and Fristoe, 1995; Briggs, Raz, and Marks, 1999, 2001; Raz, Briggs, Marks, and Acker, 1999; Rainville, Marchand, and Passini, 2002). However, very few studies have directly compared both types of transformation, and at this point, it is not clear whether there are specific deficits to one or more reference frames or transformation operations or whether age-related changes in visuospatial imagery reflect more general processes, such as attention and memory, or general cognitive slowing (Herman and Coyne, 1980; Inagaki et al., 2002).

In addition to further investigation of variability across individuals within the human species, it would be valuable to examine differences in spatial transformations across species. The multiple systems framework predicts that different species should have different sorts of abilities for imagined spatial transformations, depending on their ecological niche and evolutionary history. For example, sedentary species should have limited ability to imagine perspective transformations, and aquatic species should be better than terrestrial species at imagining perspective transformations in planes other than the horizontal. There have been a few studies of spatial transformations in nonhuman animals. Studies of pigeons provide mixed results but suggest that they may have the ability to perform object-based transformations (Hollard and Delius, 1982; Cook and Katz, 1999). Electrophysiological recordings in the monkey have identified a continuous neural transformation during the planning of arm movements, but it is not clear whether this reflects an object-based transformation or motor simulation (Georgopoulos, Lurito, Petrides, Schwarts, and Massey, 1989). Experimental studies focused on dissociating object-based and perspective transformations in nonhuman species would be of signal value.

The study of spatial reasoning would benefit from further neuroimaging studies aimed at selectively measuring the neural correlates of particular proposed computational processes. Research designs to achieve this goal will likely depend on event-related imaging methods (Buckner and Braver, 1999), which allow the experimenter to parametrically manipulate features such as stimulus orientation, size, distance, and discriminability. The design of experiments that can effectively isolate components of mental imagery within a complex reasoning task is a major challenge.

Finally, in our view the long-term goal of research in this area should be the formulation and testing of models that integrate the different types of reference frame updating reviewed here. We have reviewed evidence that the systems involved in updating effector-centered representations interact with those that update objectcentered representations. We believe that there are likely to be more of this sort of interaction, and these interactions have not been well characterized to date. To account for how people can plan actions that involve moving in the world while other objects are in motion and interacting with those objects, scientists will have to face up to the complexity of these interactions. 


\section{NOTES}

1. When used for this purpose, object-based reference frames are also sometimes called intrinsic reference frames.

2.In previous articles, we and our colleagues have referred to perspective transformations as egocentric perspective transformations (Zacks et al., 1999; Zacks, Mires, et al., 2002; Zacks, Ollinger et al., 2002). The present terminology is intended to clarify the relationship between perspective transformations and effector-based transformations, which denote the updating of different egocentric reference frames.

3.To identify relevant studies, we performed searches in the PsycInfo and MedLine databases for articles associated with the keyword mental rotation and any of the following keywords: PET, positron emission tomography, fMRI, MRI, or neuroimaging. This search returned 71 articles. Of these, 29 were excluded because they were not peerreviewed journal articles, did not report a functional neuroimaging study of mental rotation, or did not report foci of brain activity in a standardized coordinate system. For the remaining articles, reported foci of activity were converted to the stereotactic coordinate system of Talairach and Tournoux (1988) and entered into a database. When an article reported multiple analyses that reused the same degrees of freedom, the most tightly controlled task comparison was used. A total of 151 activation foci, 109 transformation-specific foci, and 42 omnibus foci were identified. The reported foci were converted to probability maps using the method described by Turkeltaub, Eden, Jones, and Zeffiro (2002). The maps were projected onto the cortical surface using the CARET software package (Van Essen et al., 2001), and thresholded to control the voxel-wise false positive rate at $p=.001$, using the Monte Carlo method described by Turkeltaub et al. (2002).

4.Aguirre and D'Esposito (1999) also discuss the importance of recognition of landmarks and of long-term memory, for navigation, but those processes bear little on visuospatial transformations.

\section{REFERENCES}

Aguirre, G. K., \& D’Esposito, M. (1999). Topographical disorientation: A synthesis and taxonomy. Brain, 122, 1613-1628.

Amorim, M.-A., \& Stucchi, N. (1997). Viewer- and object-centered mental explorations of an imagined environment are not equivalent. Cognitive Brain Research, 5, 229-239.

Aubrey, J. B., \& Dobbs, A. R. (1990). Age and sex differences in the mental realignment of maps. Experimental Aging Research, 16, 133139 .

Avraamides, M. N., Klatzky, R. L., Loomis, J. M., \& Golledge, R. G. (2004). Use of cognitive versus perceptual heading during imagined locomotion depends on the response mode. Psychological Science, 15, 403-408.

Awh, E., Jonides, J., Smith, E. E., Buxton, R. B., Frank, L. R., Love, T., et al. (1999). Rehearsal in spatial working memory: Evidence from neuroimaging. Psychological Science, 10, 433-437.

Briggs, S. D., Raz, N., \& Marks, W. (1999). Age-related deficits in generation and manipulation of mental images: The role of sensorimotor speed and working memory. Psychology E Aging, 14, 427-435.

Briggs, S. D., Raz, N., \& Marks, W. (2001). Age-related deficits in generation and manipulation of mental images: I. The role of sensorimotor speed and working memory [Correction to Briggs et al.]. (1999). Psychology E Aging, 16, 449.

Brockmole, J. R., \& Wang, R. F. (2003). Changing perspective within and across environments. Cognition, 87, B59-B67.

Buckner, R. L., \& Braver, T. S. (1999). Event related functional MRI. In P. Bandettini, \& C. Moonen (Eds.), Functional MRI (pp. 441450). Germany: Springer-Verlag.

Bundesen, C., \& Larsen, A. (1975). Visual transformation of size. Journal of Experimental Psychology: Human Perception $\mathcal{E}$ Performance, 1, 214-220.

Butters, N., Barton, M., \& Brody, B. A. (1970). Role of the right parietal lobe in the mediation of cross-modal associations and reversible operations. Cortex, 6, 174-190.

Carpenter, M., \& Proffitt, D. R. (2001). Comparing viewer and array mental rotations in different planes. Memory and Cognition, 29, 441-448.
Colby, C. L. (1998). Action-oriented spatial reference frames in cortex. Neuron, 20, 15-24.

Cook, R. G., \& Katz, J. S. (1999). Dynamic object perception by pigeons. Journal of Experimental Psychology: Animal Behavior Processes, 25, 194-210.

Cooper, L. A., \& Shepard, R. N. (1973). The time required to prepare for a rotated stimulus. Memory $\mathcal{E}$ Cognition, 1, 246-250.

Cooper, L. A., \& Shepard, R. N. (1975). Mental transformations in identification of left and right hands. Journal of Experimental Psychology-Human Perception and Performance, 104, 48-56.

Corbetta, M. \& Shulman, G. L. (1998). Human cortical mechanisms of visual attention during orienting and search. Philosophical Transactions of the Royal Society of London B: Biological Sciences, 353, 1353-1362.

Creem, S. H., Downs, T. H., Wraga, M., Proffitt, D. R., \& Downs, J. H., III. (2001). An fMRI study of imagined self-rotation. Cognitive, Affective \& Behavioral Neuroscience, 1, 239-249.

Creem, S. H., Wraga, M., \& Proffitt, D. R. (2001). Imagining physically impossible self-rotations: Geometry is more important than gravity. Cognition, 81, 41-64.

Creem-Regehr, S. H. (2003). Updating space during imagined selfand array translations. Memory $\mathcal{E}$ Cognition, 31, 941-92.

Culham, J. C., Brandt, S. A., Cavanagh, P., Kanwisher, N. G., Dale, A. M., \& Tootell, R. B. (1998). Cortical fMRI activation produced by attentive tracking of moving targets. Journal of Neurophysiology, $80,2657-2670$.

De Renzi, E. (1982). Disorders of space exploration and cognition. Chichester, United Kingdom: John Wiley and Sons.

Decety, J. (1996). Neural representations for action. Reviews in the Neurosciences, 7, 285-297.

Decety, J. (2002). Neurophysiological evidence for simulation of action. In J. Dokic, \& J. Proust (Eds.), Simulation and knowledge of action (pp. 53-72). Lyon, France: John Benjamins.

Deiber, M. P., Ibanez, V., Honda, M., Sadato, N., Raman, R., \& Hallett, M. (1998). Cerebral processes related to visuomotor imagery and generation of simple finger movements studied with positron emission tomography. NeuroImage, 7, 73-85.

Denis, M., \& Kosslyn, S. M. (1999). Scanning visual mental images: A window on the mind. Cahiers de Psychologie Cognitive/Current Psychology of Cognition, 18, 409-465.

Dror, I. E., \& Kosslyn, S. M. (1994). Mental imagery and aging. Psychology and Aging, 9, 90-102.

Dum, R. P., \& Strick, P. L. (2002). Motor areas in the frontal lobe of the primate. Physiology and Behavior, 77, 677-682.

Ekstrom, A. D., Kahana, M. J., Caplan, J. B., Fields, T. A., Isham, E. A., Newman, E. L., et al. (2003). Cellular networks underlying human spatial navigation. Nature, 425, 184-187.

Farah, M. J. (1989). The neuropsychology of mental imagery. In F. Boller, \& J. Grafman (Eds.), Handbook of Neuropsychology (pp. 395413). Amsterdam: Elsevier.

Farrell, M. J. (1996). Topographical disorientation. Neurocase, 2, 509520.

Farrell, M.J., \& Robertson, I. H. (1998). Mental rotation and the automatic updating of body-centered spatial relationships. Journal of Experimental Psychology-Learning, Memory and Cognition, 24, 227-233.

Féry, Y.-A., Magnac, R., \& Isrâ̂l, I. (2004). Commanding the direction of passive whole-body rotations facilitates egocentric spatial updating. Cognition, 91, B1-B10.

Finke, R. A. (1985). Theories relating mental imagery to perception. Psychological Bulletin, 98, 236-259.

Finke, R. A., \& Shepard, R. N. (1986). Visual functions of mental imagery. In K. R. Boff, L. Kaufman, \& J. P. Thomas (Eds.), Handbook of perception and human performance (pp. 1-55). New York: John Wiley \& Sons.

Flavell, J. H., Everett, B. A., Croft, K., \& Flavell, E. R. (1981). Young children's knowledge about visual perception: Further evidence for the Level 1-Level 2 distinction. Developmental Psychology, 17, 99-103.

Flicker, C., Ferris, S. H., Crook, T., Reisberg, B., \& Bartus, R. T. (1988). Equivalent spatial-rotation deficits in normal aging and Alzheimer's disease. Journal of Clinical E Experimental Neuropsychology, 10, 387-399. 
Franklin, N., \& Tversky, B. (1990). Searching imagined environments. Journal of Experimental Psychology: General, 119, 63-76.

Funt, B. V. (1983). A parallel-process model of mental rotation. Cognitive Science, 7, 67-93.

Gardner, H. (1985). The mind's new science: A history of the cognitive revolution. New York: Basic Books.

Geary, D. C. (1998). Male, female: The evolution of human sex differences. Washington, DC: American Psychological Association.

Georgopoulos, A. P., Lurito, J. T., Petrides, M., Schwartz, A. B., \& Massey, J. T. (1989). Mental rotation of the neuronal population vector. Science, 243, 234-236.

Graziano, M. S. A., \& Gross, C. G. (1994). Mapping space with neurons. Current Directions in Psychological Science, 3, 164-167.

Grézes, J., \& Decety, J. (2001). Functional anatomy of execution, mental simulation, observation, and verb generation of actions: A meta-analysis. Human Brain Mapping, 12, 1-19.

Hale, S., Lima, S. D., \& Myerson, J. (1991). General cognitive slowing in the nonlexical domain: An experimental validation. Psychology and Aging, 6, 512-521.

Hale, S., Myerson, J., Faust, M., \& Fristoe, N. (1995). Converging evidence for domain-specific slowing from multiple nonlexical tasks and multiple analytic methods. Journal of Gerontology B: Psychological Sciences and Social Sciences, 50, P202-P211.

Harris, I. M., \& Miniussi, C. (2003). Parietal lobe contribution to mental rotation demonstrated with rTMS. Journal of Cognitive Neuroscience, 15, 1-9.

Hegarty, M., \& Kozhevnikov, M. (1999). Spatial reasoning abilities, working memory, and mechanical reasoning. In J. S. Gero \& B. Tversky (Eds.), Visual and spatial reasoning in design (pp. 1-19). Sydney, Australia: Key Center of Design Computing and Cognition.

Hegarty, M., \& Waller, D. (2004). A dissociation between mental rotation and perspective-taking abilities. Intelligence, 32, 175-191.

Herman, J. F., \& Coyne, A. C. (1980). Mental manipulation of spatial information in young and elderly adults. Developmental Psychology, $5,537-538$.

Hertzog, C., Vernon, M. C., \& Rypma, B. (1993). Age differences in mental rotation task performance: The influence of speed/ accuracy tradeoffs. Journal of Gerontology, 48, P150-P156.

Hollard, V. D., \& Delius, J. D. (1982). Rotational invariance in visual pattern recognition by pigeons and humans. Science, 218, 804-806.

Huttenlocher, J., \& Presson, C. C. (1973). Mental rotation and the perspective problem. Cognitive Psychology, 4, 277-299.

Huttenlocher, J., \& Presson, C. C. (1979). The coding and transformation of spatial information. Cognitive Psychology, 11, 375-394.

Inagaki, H., Meguro, K., Shimada, M., Ishizaki, J., Okuzumi, H., \& Yamadori, A. (2002). Discrepancy between mental rotation and perspective-taking abilities in normal aging assessed by Piaget's three-mountain task. Journal of Clinical E् Experimental Neuropsychology, 24, 18-25.

Isaac, A. R., \& Marks, D. F. (1994). Individual-differences in mentalimagery experience-developmental-changes and specialization. British Journal of Psychology, 85, 479-500.

Jeannerod, M. (1995). Mental imagery in the motor context. Neuropsychologia, 33, 1419-1432.

Jeannerod, M., \& Frak, V. (1999). Mental imaging of motor activity in humans. Current Opinion in Neurobiology, 9, 735-739.

Just, M. A., \& Carpenter, P. A. (1985). Cognitive coordinate systems: Accounts of mental rotation and individual differences in spatial ability. Psychological Review, 92, 137-172.

Kail, R. (1985). Development of mental rotation: A speed-accuracy study. Journal of Experimental Child Psychology, 40, 181-192.

Kail, R., Pellegrino, J., \& Carter, P. (1980). Developmental changes in mental rotation. Journal of Experimental Child Psychology, 29, 102-116.

Kimura, D. (1999). Sex and cognition. Cambridge, MA: MIT Press.

Klatzky, R. L., Loomis, J. M., Beall, A. C., Chance, S. S., \& Golledge, R. G. (1998). Spatial updating of self-position and orientation during real, imagined, and virtual locomotion. Psychological Science, 9, 293-298.

Köhler, W. (1929). Gestalt psychology. New York: H. Liveright.

Kosslyn, S. M. (1980). Image and mind. Cambridge, MA: Harvard University Press.
Kosslyn, S. M. (1981). The medium and the message in mental imagery: A theory. Psychological Review, 88, 46-66.

Kosslyn, S. M. (1994). Image and brain: the resolution of the imagery debate. Cambridge, MA: MIT Press.

Kosslyn, S. M., Ball, T. M., \& Reiser, B. J. (1978). Visual images preserve metric spatial information: Evidence from studies of image scanning. Journal of Experimental Psychology: Human Perception $\mathcal{E}$ Performance, 4, 47-60.

Kosslyn, S. M., DiGirolamo, G. J., Thompson, W. L., \& Alpert, N. M. (1998). Mental rotation of objects versus hands: Neural mechanisms revealed by positron emission tomography. Psychophysiology, $35,151-161$

Kosslyn, S. M., \& Pomerantz, J. R. (1977). Imagery, propositions, and the form of internal representations. Cognitive Psychology, 9, 52-76.

Kosslyn, S. M., Thompson, W. L., Wraga, M., \& Alpert, N. M. (2001). Imagining rotation by endogenous versus exogenous forces: Distinct neural mechanisms. NeuroReport, 12, 2519-2525.

Kozhevnikov, M., \& Hegarty, M. (2001). A dissociation between object manipulation spatial ability and spatial orientation ability. Memory and Cognition, 29, 745-756.

Larsen, A. (1985). Pattern matching: Effects of size ratio, angular difference in orientation, and familiarity. Perception E Psychophysics, $38,63-68$.

Larsen, A., \& Bundesen, C. (1978). Size scaling in visual pattern recognition. Journal of Experimental Psychology: Human Perception E Performance, 4, 1-20.

Larsen, A., \& Bundesen, C. (1998). Effects of spatial separation in visual pattern matching: Evidence on the role of mental translation. Journal of Experimental Psychology: Human Perception $\mathcal{E} \mathcal{O}$ Performance, 24, 719-731.

Leutgeb, S., Leutgeb, J. K., Barnes, C. A., Moser, E. I., McNaughton, B. L., \& Moser, M.-B. (2005). Independent codes for spatial and episodic memory in hippocampal neuronal ensembles. Science, 309, 619-623.

Levinson, S. C. (1996). Frames of reference and Molyneux's question: Crosslinguistic evidence. Language, speech, and communication. In P. Bloom \& M. A. Peterson (Eds.), Language and space (pp. 109169). Cambridge, MA: MIT Press.

Likert, R., \& Quasha, W. H. (1948). The revised Minnesota paper form board test. New York: The Psychological Corporation.

McCloskey, M. (2001). Spatial representation in mind and brain. In B. Rapp (Ed.), The handbook of cognitive neuropsychology: What deficits reveal about the human mind (pp. 101-132). Philadelphia: Psychology Press.

Michelon, P., Vettel, J. M., \& Zacks, J. M. Somatotopy during imagined and prepared movements. Manuscript submitted for publication.

Michelon, P., \& Zacks, J. M. (in press). Two kinds of visual perspectivetaking. Perception and Psychophysics.

Miller, G. A. (1962). Psychology, the science of mental life. New York: Harper \& Row.

Mou, W., \& McNamara, T. P. (2002). Intrinsic frames of reference in spatial memory. Journal of Experimental Psychology: Learning, Memory, EO Cognition, 28, 162-170.

Mou, W., McNamara, T. P., Valiquette, C. M., \& Rump, B. (2004). Allocentric and egocentric updating of spatial memories. Journal of Experimental Psychology: Learning, Memory and Cognition, 30, 142157.

Newcombe, F., \& Ratcliff, G. (1989). Disorders of visuospatial analysis. In F. Boller, \& J. Grafman (Eds.), Handbook of neuropsychology (pp. 333-356). Amsterdam: Elsevier.

Newcombe, N. (1989). The development of spatial perspective taking. Advances in Child Development and Behavior, 22, 203-247.

Newcombe, N., \& Huttenlocher, J. (1992). Children's early ability to solve perspective-taking problems. Developmental Psychology, 28, 635-643.

Newcombe, N. S. (2002). Spatial cognition. In H. Pashler \& D. Medin (Eds.), Steven's handbook of experimental psychology, Vol. 2 (pp. 11363). San Francisco: Jossey-Bass.

O'Keefe, J., \& Nadel, L. (1978). The hippocampus as a cognitive map. Oxford, United Kingdom: Oxford University Press. 
Pani, J. R. (1993). Limits on the comprehension of rotational motion: Mental imagery of rotations with oblique components. Perception, 22, 785-808.

Parsons, L. M. (1987a). Imagined spatial transformation of one's body. Journal of Experimental Psychology: General, 116, 172-191.

Parsons, L. M. (1987b). Visual discrimination of abstract mirrorreflected three-dimensional objects at many orientations. Perception $\mathcal{E}$ Psychophysics, 42, 49-59.

Parsons, L. M. (1994). Temporal and kinematic properties of motor behavior reflected in mentally simulated action. Journal of Experimental Psychology: Human Perception E Performance, 20, 709-730.

Parsons, L. M., Fox, P. T., Downs, J. H., Glass, T., Hirsch, T. B., Martin, C. C., et al. (1995). Use of implicit motor imagery for visual shape discrimination as revealed by PET. Nature, 375, 54-58.

Parsons, L. M., Gabrieli, J. D., Phelps, E. A., \& Gazzaniga, M. S. (1998). Cerebrally lateralized mental representations of hand shape and movement. Journal of Neuroscience, 18, 6539-6548.

Paus, T. (1996). Location and function of the human frontal eyefield: a selective review. Neuropsychologia, 34, 475-483.

Penfield, W., \& Rasmussen, T. (1950). The cerebral cortex of man. New York: Macmillan.

Philbeck, J. W., Behrmann, M., Levy, L., Potolicchio, S. J., \& Caputy, A. J. (2004). Path integration deficits during linear locomotion after human medial temporal lobectomy. Journal of Cognitive Neuroscience, $16,510-520$.

Piaget, J., \& Inhelder, B. (1956). The child's conception of space. London: Routledge \& K. Paul.

Picard, N., \& Strick, P. L. (2001). Imaging the premotor areas. Current Opinion in Neurobiology, 11, 663-672.

Pinker, S. (1988). A computational theory of the mental imagery medium. In M. Denis \& J. Engelkamp (Eds.), Cognitive and neuropsychological approaches to mental imagery (pp. 17-32). Dordrecht, the Netherlands: Netherlandsshing.

Presson, C. C. (1980). Spatial egocentrism and the effect of an alternate frame of reference. Journal of Experimental Child Psychology, 29, 391-402.

Presson, C. C. (1982). Strategies in spatial reasoning. Journal of Experimental Psychology: Learning, Memory, E् Cognition, 8, 243-251.

Presson, C. C., \& Montello, D. R. (1994). Updating after rotational and translational body movements: Coordinate structure of perspective space. Perception, 23, 1447-1455.

Pylyshyn, Z. (2001). Is the imagery debate over? If so, what was it about? In E. Dupoux (Ed.), Language, brain, and cognitive development: Essays in honor of Jacques Mehler (pp. 59-83). Cambridge, MA: The MIT Press.

Pylyshyn, Z. W. (1973). What the mind's eye tells the mind's brain: A critique of mental imagery. Psychological Bulletin, 80, 1-24.

Pylyshyn, Z. W. (1981). The imagery debate: Analogue media versus tacit knowledge. Psychological Review, 88, 16-45.

Pylyshyn, Z. W. (2002). Mental imagery: In search of a theory. Behavioral and Brain Sciences, 25, 157-182.

Pylyshyn, Z. W. (2003). Return of the mental image: Are there really pictures in the brain? Trends in Cognitive Science, 7, 113-118.

Rainville, C., Marchand, N., \& Passini, R. (2002). Performances of patients with a dementia of the Alzheimer type in the standardized road-map test of direction sense. Neuropsychologia, 40, 567-573.

Raz, N., Briggs, S. D., Marks, W., \& Acker, J. D. (1999). Age-related deficits in generation and manipulation of mental images: II. The role of dorsolateral prefrontal cortex. Psychology E Aging, 14, 436-444.

Richter, W., Ugurbil, K., Georgopoulos, A., \& Kim, S. G. (1997). Timeresolved fMRI of mental rotation. Neuroreport, 8, 3697-3702.

Rieser, J. J. (1989). Access to knowledge of spatial structure at novel points of observation. Journal of Experimental Psychology: Learning, Memory, EO Cognition, 15, 1157-1165.

Rock, I. (1974). The perception of disoriented figures. Scientific American, 230, 78-85.

Rock, I., Wheeler, D., \& Tudor, L. (1989). Can we imagine how objects look from other viewpoints. Cognitive Psychology, 21, 185-210.

Rosenbaum, R. S., Priselac, S., Köhler, S., Black, S. E., Gao, F., Nadel, L., et al. (2000). Remote spatial memory in an amnesic person with extensive bilateral hippocampal lesions. Nature Neuroscience, 3, 1044-1048.
Salatas, H., \& Flavell, J. H. (1976). Perspective taking: The development of two components of knowledge. Child Development, 47, 103109.

Schultz, D. P. (1975). A history of modern psychology. New York: Academic Press.

Schwartz, D. L., \& Holton, D. L. (2000). Tool use and the effect of action on the imagination. Journal of Experimental Psychology: Learning, Memory, E Cognition, 26, 1655-1665.

Sekiyama, K. (1982). Kinesthetic aspects of mental representations in the identification of left and right hands. Perception $\mathcal{E}$ Psychophysics, 32, 89-95.

Semmes, J., Weinstein, S., Ghent, L., \& Teuber, H.-L. (1955). Spatial orientation in man after cerebral injury: I. Analyses by locus of lesion. Journal of Psychology, 39, 227-244.

Semmes, J., Weinstein, S., Ghent, L., \& Teuber, H.-L. (1963). Correlates of impaired orientation in personal and extrapersonal space. Brain, 86, 747-772.

Shelton, A. L., \& McNamara, T. P. (2001). Systems of spatial reference in human memory. Cognitive Psychology, 43, 274-310.

Shepard, R. N. (1994). Perceptual-cognitive universals as reflections of the world. Psychonomic Bulletin and Review, 1, 2-28.

Shepard, R. N., \& Cooper, L. A. (1982). Mental images and their transformations. Cambridge, MA: MIT Press.

Shepard, R. N., \& Metzler, J. (1971). Mental rotation of threedimensional objects. Science, 171, 701-703.

Sholl, M.J., \& Bartels, G. P. (2002). The role of self-to-object updating in orientation-free performance on spatial-memory tasks. Journal of Experimental Psychology: Learning, Memory, E Cognition, 28, 422436.

Simons, D. J., \& Wang, R. F. (1998). Perceiving real-world viewpoint changes. Psychological Science, 9, 315-320.

Sirigu, A., \& Duhamel, J. R. (2001). Motor and visual imagery as two complementary but neurally dissociable mental processes. Journal of Cognitive Neuroscience, 13, 910-919.

Sirigu, A., Duhamel, J. R., Cohen, L., Pillon, B., Dubois, B., \& Agid, Y. (1996). The mental representation of hand movements after parietal cortex damage. Science, 273, 1564-1568.

Snyder, L. H., Batista, A. P., \& Andersen, R. A. (1997). Coding of intention in the posterior parietal cortex. Nature, 386, 167-170.

Tagaris, G. A., Kim, S.-G., Strupp, J. P., Andersen, P., Ugurbil, K., \& Georgopoulos, A. P. (1997). Mental rotation studied by functional magnetic resonance imaging at high field (4 Tesla): Performance and cortical activation. Journal of Cognitive Neuroscience, 9, 419-432.

Talairach, J., \& Tournoux, P. (1988). Co-planar stereotaxic atlas of the human brain: 3-dimensional proportional system, an approach to cerebral imaging. Stuttgart, Germany: G. Thieme.

Teng, E., \& Squire, L. R. (1999). Memory for places learned long ago is intact after hippocampal damage. Nature, 400, 687-677.

Thayer, Z. C., Johnson, B. W., Corballis, M. C., \& Hamm, J. P. (2001). Perceptual and motor mechanisms for mental rotation of human hands. NeuroReport, 12, 3433-3437.

Turkeltaub, P. E., Eden, G. F., Jones, K. M., \& Zeffiro, T. A. (2002). Meta-analysis of the functional neuroanatomy of single-word reading: method and validation. NeuroImage, 16, 765-780.

Van Essen, D. C., Drury, H. A., Dickson, J., Harwell, J., Hanlon, D., \& Anderson, C. H. (2001). An integrated software suite for surfacebased analyses of cerebral cortex. Journal of the American Medical Informatics Association, 8, 443-459.

van Lier, R. (2003). Differential effects of object orientation on imaginary object/viewer transformations. Psychonomic Bulletin $\mathcal{E}$ Review, 10, 455-461.

Vingerhoets, G., de Lange, F. P., Vandemaele, P., Deblaere, K., \& Achten, E. (2002). Motor imagery in mental rotation: an fMRI study. NeuroImage, 17, 1623-1633.

Wang, R. X. F., \& Simons, D. J. (1999). Active and passive scene recognition across views. Cognition, 70, 191-210.

Wexler, M., Kosslyn, S. M., \& Berthoz, A. (1998). Motor processes in mental rotation. Cognition, 68, 77-94.

Wohlschläger, A. (2001). Mental object rotation and the planning of hand movements. Perception and Psychophysics, 63, 709-718. 
Wohlschläger, A., \& Wohlschläger, A. (1998). Mental and manual rotation. Journal of Experimental Psychology: Human Perception $\mathcal{E} \mathcal{O}$ Performance, 24, 397-412.

Wraga, M. (2003). Thinking outside the body: An advantage for spatial updating during imagined versus physical self-rotation. Journal of Experimental Psychology: Learning, Memory and Cognition, 29, 993-1005.

Wraga, M., Creem, S. H., \& Proffitt, D. R. (1999). The influence of spatial reference frames on imagined object- and viewer rotations. Acta Psychologica, 102, 247-264.

Wraga, M., Creem, S. H., \& Proffitt, D. R. (2000). Updating scenes after object- and viewer-rotations. Journal of Experimental Psychology: Learning, Memory, and Cognition, 26, 151-168.

Wraga, M., Creem, S. H.-R., \& Proffitt, D. R. (2004). Spatial updating of virtual displays during self- and display rotation. Memory and Cognition, 32, 399-415.

Wraga, M., Shephard, J. M., Church, J. A., Inati, S., \& Kosslyn, S. M. (2005). Imagined rotations of self versus objects: An fMRI study. Neuropsychologia, 43, 1351-1361.

Wraga, M., Thompson, W. L., Alpert, N. M., \& Kosslyn, S. M. (2003). Implicit transfer of motor strategies in mental rotation. Brain and Cognition, 52, 135-143.
Zacks, J. M. Functional neuroimaging of mental rotation: A metaanalysis. Unpublished manuscript.

Zacks, J. M., Gilliam, F., \& Ojemann, J. G. (2003). Selective disturbance of mental rotation by cortical stimulation. Neuropsychologia, 41, 1659-1667.

Zacks, J. M., Mires, J., Tversky, B., \& Hazeltine, E. (2002) . Mental spatial transformations of objects and perspective. Spatial Cognition $\mathcal{E}$ Computation, 2, 315-322.

Zacks, J. M., Ollinger, J. M., Sheridan, M. A., \& Tversky, B. (2002). A parametric study of mental spatial transformations of bodies. NeuroImage, 16, 857-872.

Zacks, J. M., Rypma, B., Gabrieli, J., Tversky, B., \& Glover, G. (1999). Imagined transformations of bodies: An fMRI study. Neuropsychologia, 37, 1029-1040.

Zacks, J. M., \& Shelton, A. L. (2003). Imagined transformations of bodies and rooms. Abstracts of the Psychonomic Society, 8, 31.

Zacks, J. M., \& Tversky, B. (in press). Multiple systems for spatial imagery: Transformations of objects and bodies. Spatial Cognition $\mathcal{E}$ Computation.

Zacks, J. M., Vettel, J. M., \& Michelon, P. (2003). Imagined viewer and object rotations dissociated with event-related fMRI. Journal of Cognitive Neuroscience, 15, 1002-1018. 